\title{
Optimal Shrinkage Estimation of Variances with Applications to Microarray Data Analysis
}

\author{
Tiejun Tong and Yuedong WANG *
}

May 27, 2005

\begin{abstract}
Microarray technology allows a scientist to study genome-wide patterns of gene expression. Thousands of individual genes are measured with relatively small number of replications which poses challenges to traditional statistical methods. In particular, the gene-specific estimators of variances are not reliable and gene-by-gene tests have low power. In this paper we propose a family of shrinkage estimators for variances raised to a fixed power. We derive optimal shrinkage parameters under both Stein and the squared loss functions. Our results show that the standard sample variance is inadmissible under either loss functions. We propose several estimators for the optimal shrinkage parameters and investigate their asymptotic properties under two scenarios: large number of replications and large number of genes. We conduct simulations to evaluate the finite sample performance of the data-driven optimal shrinkage estimators and compare them with some existing methods. We construct $F$-like statistics using these shrinkage variance estimators and apply them to detect differentially expressed genes in a microarray experiment. We also conduct simulations to evaluate performance of these $F$-like statistics and compare them with some existing methods.
\end{abstract}

Key words and phrases: F-like statistic, gene expression data, inadmissibility, James-Stein shrinkage estimator, loss function.

\section{INTRODUCTION}

The development of microarray technology has revolutionized the study of molecular biology and become a standard tool in genomics research. Instead of working on a gene-by-gene basis, microarray technology allows the scientists to view the expression of thousands of genes from an experimental sample simultaneously (Nguyen, Arpat, Wang and Carroll 2002, Leung and

*Tiejun Tong (email: tong@pstat.ucsb.edu) is Ph.D candidate, and Yuedong Wang (email: yuedong@pstat.ucsb.edu) is Professor, Department of Statistics and Applied Probability, University of California, Santa Barbara, California 93106. This research was supported by NIH Grant R01 GM58533. The authors thank Dr. Xiangqin Cui in the Jackson Laboratory for answering many technical questions about the MAANOVA package. 
Cavalieri 2003). Due to the cost, it is common that thousands of genes are measured with a small number of replications (Lönnstedt and Speed 2002, Kendziorski, Newton, Lan and Could 2003). As a consequence, we are facing with a "large $G$, small $n$ " paradigm, where $G$ is total number of genes and $n$ is the number of replications.

The standard gene-specific estimators of variances are unreliable due to the relatively small number of replications. Consequently, the commonly used statistical methods such as t-test or F-test for detecting differentially expressed genes on a gene-by-gene basis have low power (Callow, Dudoit, Gong, Speed and Rubin 2000). On the other hand, the assumption that variances are equal for all genes is unlikely to be true. Thus, tests based on a pooled common variance estimator for all genes are at the risk of generating misleading results (Wright and Simon 2003, Cui, Hwang, Qiu, Blades and Churchill 2005).

A number of approaches to improving variance estimation and hypothesis test have emerged. Kamb and Ramaswami (2001) suggested a simple regression estimation of local variances. Storey and Tibshirani (2003) added a small constant to the gene-specific variance estimators in their SAM $t$-test in order to stabilize the small variances. Lin, Nadler, Attie and Yandell (2003) proposed a data-adapted robust estimator of array error based on a smoothing spline and standardized local median absolute deviation. Jain, Thatte, Braciale, Ley, O'Connell and Lee (2003) proposed a local-pooled-error estimation procedure which borrows strength from genes in local intensity regions for estimating array error variability. Baldi and Long (2001) proposed a regularized $t$-test by replacing the usual variance estimator with a Bayesian estimator. Lönnstedt and Speed (2002) proposed an empirical Bayes approach that combines information across genes. Kendziorski et al. (2003) extended the empirical Bayes method using hierarchical gamma-gamma and lognormal-normal models.

Cui and Churchill (2003) compared three variance estimators: the gene-specific estimator, the pooled estimator across genes, and the hybrid estimator as the averages of the gene-specific and the pooled estimators. Cui et al. (2005) proposed James-Stein type shrinkage estimator for variances (referred to as the CHQBC estimator in the remainder of this article). Compared to some existing tests, they showed that the $F$-test using the James-Stein type variance estimator has the best or nearly the best power for detecting differentially expressed genes over a wide range of situations.

The research so far has concentrated on the methodology. Little is known about the theoretical properties of various shrinkage variance estimators. The shrinkage variance estimation has a long history starting with the amazing inadmissibility result discovered by Stein (1964), where the standard sample variance is improved by a shrinkage estimator using information contained in the sample mean. Much research has been done since then (Maatta and Casella 1990, Kubokawa 1999). Most research concerned with a single variance (Brown 1968, Brewster and Zidek 1974, Kubokawa 1994) which are not applicable to microarray data analysis since the homogeneity of the variances is unlikely to be true. Some research has devoted to the shrinkage estimator of a covariance matrix (Sinha and Ghosh 1987, Perron 1990, Kubokawa and Srivastava 2003). However, all these methods required $n>G$ to ensure non-singularity of the sample covariance matrix. Therefore, these methods broke down for microarray data analysis. 
We propose new optimal shrinkage estimators in this paper. Instead of using information in the sample mean (Stein 1964), we borrow information across variances. We will show that the standard sample variance is inadmissible. Therefore, our results extend Stein's theory for multiple means (James and Stein 1961) to multiple variances. An important insight of this paper is that a better variance estimator does not necessarily lead to a more powerful test. Specifically, since the variance appears in the denominator, an F-test using an estimator of the reciprocal of the variance is more powerful than that using the reciprocal of an estimator of the variance (Section 5). We consider optimal shrinkage estimators for the parameter $\left(\sigma_{g}^{2}\right)^{t}$ where $\sigma_{g}^{2}$ is the true variance associated with gene $g, g=1, \ldots, G$, and $t$ is a fixed non-zero power. Most existing research focuses on the special case of estimating $\sigma_{g}^{2}$. We will show that our methods compare favorably to all existing methods using simulations.

Our methods and theory are general. Nevertheless, we present our methods in the framework of microarray data analysis. In Section 2, we introduce the CHQBC estimator and propose a modified version. In Section 3, we derive optimal shrinkage estimators for $\left(\sigma_{g}^{2}\right)^{t}$ under two common loss functions and show that the optimal shrinkage estimators dominate the standard gene-specific variance estimator. We also propose various estimators for the optimal shrinkage parameters and investigate their asymptotic properties under two scenarios: $n \rightarrow \infty$ with fixed $G$ and $G \rightarrow \infty$ with fixed $n$. In Section 4 , we conduct simulations to evaluate the performance of the optimal shrinkage estimators and compare them with the CHQBC estimator and the modified CHQBC estimator. In Section 5, we construct F-like statistics using our optimal shrinkage estimators to detect differentially expressed genes for a microarray data, and conduct simulations to evaluate and compare performances of these $F$-like statistics. We conclude this article in Section 6 with a brief discussion.

\section{CHQBC ESTIMATOR AND ITS MODIFICATION}

Let $G(G \geq 3)$ be the number of genes, $X_{g} \sim \sigma_{g}^{2} \chi_{\nu}^{2}$ and $X_{g}$ 's are mutually independent. $X_{g}$ 's are usually the residual sum of squares and $\nu$ is the degree of freedom. Consider the following transformation

$$
X_{g}^{\prime}=\ln \sigma_{g}^{2}+\epsilon_{g}^{\prime},
$$

where $X_{g}^{\prime}=\ln \left(X_{g} / \nu\right)-m, \epsilon_{g}^{\prime}=\ln \left(\chi_{\nu}^{2} / \nu\right)-m$ and $m=\mathrm{E}\left(\ln \left(\chi_{\nu}^{2} / \nu\right)\right)$. For simplicity, $\chi_{\nu}^{2}$ also denotes the random variable which follows a Chi-squared distribution with $\nu$ degrees of freedom. Applying the James-Stein shrinkage method to $X_{g}^{\prime}$ (Lindley 1962) and then transform back to the original scale, Cui et al. (2005) proposed the following CHQBC estimator

$$
\tilde{\sigma}_{g}^{2}=\left(\prod_{g=1}^{G}\left(X_{g} / \nu\right)^{1 / G}\right) B \times \exp \left[\left(1-\frac{(G-3) V}{\sum\left(\ln X_{g}-\overline{\ln X_{g}}\right)^{2}}\right)_{+} \times\left(\ln X_{g}-\overline{\ln X_{g}}\right)\right],
$$

where $V=\operatorname{var}\left(\epsilon_{g}^{\prime}\right), \overline{\ln X_{g}}=\sum_{g=1}^{G} \ln \left(X_{g}\right) / G$ and $B=\exp (-m)$.

Let $Z_{g}=X_{g} / \nu, Z_{\text {pool }}=\prod_{g=1}^{G} Z_{g}^{1 / G}$ and $\hat{\alpha}_{0}=1-\left(1-(G-3) V / \sum\left(\ln X_{g}-\overline{\ln X_{g}}\right)^{2}\right)_{+}$. It is easy to check that the CHQBC estimator (1) can be rewritten as

$$
\tilde{\sigma}_{g}^{2}=B\left(Z_{\text {pool }}\right)^{\alpha}\left(Z_{g}\right)^{1-\alpha}
$$


with $\alpha=\hat{\alpha}_{0}$. Note that when $\sigma_{g}^{2}=\sigma^{2}$ for all $g, \mathrm{E}\left(Z_{\text {pool }}\right)=\sigma^{2} / B$. That is, $B Z_{\text {pool }}$ is an unbiased estimator of $\sigma^{2}$ when $\sigma_{g}^{2}=\sigma^{2}$ for all $g$. On the other hand, $Z_{g}$ is an unbiased estimator of $\sigma_{g}^{2}$. Therefore, it is reasonable to consider the following combination of two unbiased estimators

$$
\hat{\sigma}_{g}^{2}=\left(B Z_{\text {pool }}\right)^{\alpha}\left(Z_{g}\right)^{1-\alpha}, \quad 0 \leq \alpha \leq 1
$$

When necessary, the dependence of $\hat{\sigma}_{g}^{2}$ on $\alpha$ will be expressed explicitly as $\hat{\sigma}_{g}^{2}(\alpha)$. We refer to $\hat{\sigma}_{g}^{2}\left(\hat{\alpha}_{0}\right)$ as the modified CHQBC estimator. When variances $\sigma_{g}^{2}$ are similar, it is likely that $\hat{\alpha}_{0} \approx 1$ and thus $\hat{\sigma}_{g}^{2}\left(\hat{\alpha}_{0}\right) \approx \tilde{\sigma}_{g}^{2}\left(\hat{\alpha}_{0}\right)$. When $\hat{\alpha}_{0} \approx 0, \tilde{\sigma}_{g}^{2}\left(\hat{\alpha}_{0}\right) \approx B Z_{g}$ which is biased when $B \neq 1$. Simulations in Section 4.1 indicate that the modified CHQBC estimator $\hat{\sigma}_{g}^{2}\left(\hat{\alpha}_{0}\right)$ always performs better than the original CHQBC estimator $\tilde{\sigma}_{g}^{2}\left(\hat{\alpha}_{0}\right)$ for estimating $\sigma_{g}^{2}$.

The estimator $\hat{\sigma}_{g}^{2}$ has a very simple structure: it borrows information across genes by shrinking each gene-specific variance toward the bias corrected geometric mean of variances for all genes. The shrinkage parameter $\hat{\alpha}_{0}$ was obtained by applying the James-Stein method to the logarithm of sample variances which do not follow the normal distribution (Cui et al. 2005). Therefore, $\hat{\alpha}_{0}$ may not be optimal and theoretical properties of $\tilde{\sigma}_{g}^{2}$ and $\hat{\sigma}_{g}^{2}$ are unknown.

\section{OPTIMAL SHRINKAGE}

We now consider the family of shrinkage estimators $\hat{\sigma}_{g}^{2}$ in (3) with the shrinkage parameter $\alpha$ unfixed. There is no shrinkage when $\alpha=0$, and all variance estimates are shrunk to the bias corrected geometric mean when $\alpha=1$. Our goal is to find the optimal shrinkage parameter $\alpha$ under Stein loss function (James and Stein 1961)

$$
L_{1}\left(\sigma^{2}, \hat{\sigma}^{2}\right)=\hat{\sigma}^{2} / \sigma^{2}-\ln \left(\hat{\sigma}^{2} / \sigma^{2}\right)-1
$$

and the squared loss function

$$
L_{2}\left(\sigma^{2}, \hat{\sigma}^{2}\right)=\left(\hat{\sigma}^{2} / \sigma^{2}-1\right)^{2} .
$$

(4) is also called the entropy loss or Kullback-Leibler loss function (Kubokawa 1999). Though more complicated than the squared loss function, Stein loss function has some desirable properties. It is easy to see that $L_{1}\left(\sigma^{2}, \sigma^{2}\right)=L_{2}\left(\sigma^{2}, \sigma^{2}\right)=0, L_{1}\left(\sigma^{2}, \hat{\sigma}^{2}\right) \rightarrow \infty$ and $L_{2}\left(\sigma^{2}, \hat{\sigma}^{2}\right) \rightarrow 1$ as $\hat{\sigma}^{2} \rightarrow 0$, and $L_{1}\left(\sigma^{2}, \hat{\sigma}^{2}\right) \rightarrow \infty$ and $L_{2}\left(\sigma^{2}, \hat{\sigma}^{2}\right) \rightarrow \infty$ as $\hat{\sigma}^{2} \rightarrow \infty$. This means that Stein loss function penalizes gross underestimation as heavily as gross overestimation, while the squared loss function penalizes the gross underestimation less than the gross overestimation.

Throughout the remainder of this article, we assume that $Z_{g}=\sigma_{g}^{2} \chi_{\nu}^{2} / \nu, g=1, \ldots, G$, are independent random variables and $G \geq 2$. As discussed in Section 1, we will derive an optimal shrinkage estimator for $\left(\sigma_{g}^{2}\right)^{t}$ for any power $t \neq 0 . \sigma_{g}^{2}$ and $1 / \sigma_{g}^{2}$ are special cases with $t=1$ and $t=-1$. We will first generalize the estimator (3) for estimating $\left(\sigma_{g}^{2}\right)^{t}$. Define

$$
h_{n}(t)=\left(\frac{\nu}{2}\right)^{t}\left(\frac{\Gamma\left(\frac{\nu}{2}\right)}{\Gamma\left(\frac{\nu}{2}+\frac{t}{n}\right)}\right)^{n},
$$

where $\Gamma(\cdot)$ is the Gamma function. 
Lemma 1. For any non-zero $t>-\nu / 2$,

(i) $h_{1}(t) Z_{g}^{t}$ is an unbiased estimator of $\left(\sigma_{g}^{2}\right)^{t}$;

(ii) When $\sigma_{g}^{2}=\sigma^{2}$ for all $g, h_{G}(t) Z_{\text {pool }}^{t}$ is an unbiased estimator of $\left(\sigma^{2}\right)^{t}$.

The proof is straightforward. Note that $h_{1}(t) Z_{g}^{t}$ is the gene-specific estimator of $\left(\sigma_{g}^{2}\right)^{t}$. Combining (3) and Lemma 1, we now propose a family of shrinkage estimators for $\left(\sigma_{g}^{2}\right)^{t}$,

$$
\hat{\sigma}_{g}^{2 t}=\left(h_{G}(t) Z_{\text {pool }}^{t}\right)^{\alpha}\left(h_{1}(t) Z_{g}^{t}\right)^{1-\alpha}, \quad 0 \leq \alpha \leq 1 .
$$

Note that $h_{1}(1)=1$ and $h_{G}(1) \rightarrow B$ as $G \rightarrow \infty$. Therefore, when $t=1$ and $G$ is large, (7) reduces to (3). Let $\sigma_{\text {pool }}^{2}=\left(\prod_{g=1}^{G} \sigma_{g}^{2}\right)^{1 / G}, \boldsymbol{\sigma}^{2 t}=\left(\sigma_{1}^{2 t}, \cdots, \sigma_{G}^{2 t}\right)$ and $\hat{\boldsymbol{\sigma}}^{2 t}=\left(\hat{\sigma}_{1}^{2 t}, \cdots, \hat{\sigma}_{G}^{2 t}\right)$.

\subsection{Optimal Estimator under Stein Loss Function}

Under Stein loss function (4), it is easy to check that the average risk for each gene is

$$
\begin{aligned}
R_{1}\left(\boldsymbol{\sigma}^{2 t}, \hat{\boldsymbol{\sigma}}^{2 t}\right) \triangleq & \frac{1}{G} \sum_{g=1}^{G} \mathrm{E}\left(L_{1}\left(\sigma_{g}^{2 t}, \hat{\sigma}_{g}^{2 t}\right)\right) \\
= & \frac{h_{G}^{\alpha}(t) h_{1}^{1-\alpha}(t)}{h_{1}^{G-1}\left(\frac{\alpha t}{G}\right) h_{1}\left(\left(1-\alpha+\frac{\alpha}{G}\right) t\right)}\left(\sigma_{\text {pool }}^{2}\right)^{\alpha t} \frac{1}{G} \sum_{g=1}^{G}\left(\sigma_{g}^{2}\right)^{-\alpha t} \\
& -\ln \left(h_{G}^{\alpha}(t) h_{1}^{1-\alpha}(t)\right)-t \Psi\left(\frac{\nu}{2}\right)+t \ln \left(\frac{\nu}{2}\right)-1,
\end{aligned}
$$

where $t>-\nu / 2, \Psi(t)=\Gamma^{\prime}(t) / \Gamma(t)$ is the digamma function (Abramowitz and Stegun 1972), and the second equality is derived after some tedious but straightforward algebra using Lemma 1 and the fact that $\mathrm{E} \ln \left(\chi_{\nu}^{2}\right)=\Psi(\nu / 2)+\ln (2)$. Then the optimal $\alpha$ under Stein loss function $\alpha_{1}^{*}=\underset{\alpha \in[0,1]}{\operatorname{argmin}} R_{1}\left(\boldsymbol{\sigma}^{2 t}, \hat{\boldsymbol{\sigma}}^{2 t}\right)$. Denote the optimal estimator under Stein loss function as $\hat{\sigma}_{g}^{2 t}\left(\alpha_{1}^{*}\right)$. In what follows the derivatives $R_{k}^{\prime}\left(\boldsymbol{\sigma}^{2 t}, \hat{\boldsymbol{\sigma}}^{2 t}\right)$ and $R_{k}^{\prime \prime}\left(\boldsymbol{\sigma}^{2 t}, \hat{\boldsymbol{\sigma}}^{2 t}\right)$ are with respect to $\alpha, k=1,2$. We prove in Appendix 1 that

Theorem 1. For any fixed $G, \nu$ and non-zero $t>-\nu / 2, R_{1}\left(\boldsymbol{\sigma}^{2 t}, \hat{\boldsymbol{\sigma}}^{2 t}\right)$ is a strictly convex function of $\alpha$ on $[0,1]$ satisfying

(i) $\left.R_{1}^{\prime}\left(\boldsymbol{\sigma}^{2 t}, \hat{\boldsymbol{\sigma}}^{2 t}\right)\right|_{\alpha=0}<0$;

(ii) $\left.R_{1}^{\prime}\left(\boldsymbol{\sigma}^{2 t}, \hat{\boldsymbol{\sigma}}^{2 t}\right)\right|_{\alpha=1} \geq 0$, where the equality holds if and only if $\sigma_{g}^{2}=\sigma^{2}$ for all $g$;

(iii) $R_{1}^{\prime \prime}\left(\boldsymbol{\sigma}^{2 t}, \hat{\boldsymbol{\sigma}}^{2 t}\right)>0$ for all $\alpha \in[0,1]$.

It is an immediate result from Theorem 1 that

Corollary 1. For any fixed $G, \nu$ and non-zero $t>-\nu / 2$, under Stein loss function,

(i) there exists a unique $\alpha_{1}^{*}$ in $(0,1]$ which is the solution to $R_{1}^{\prime}\left(\boldsymbol{\sigma}^{2 t}, \hat{\boldsymbol{\sigma}}^{2 t}\right)=0$;

(ii) $h_{1}(t) Z_{g}^{t}$ is inadmissible for $\left(\sigma_{g}^{2}\right)^{t}$ since $\alpha_{1}^{*}>0$;

(iii) $\alpha_{1}^{*}=1$ iff $\sigma_{g}^{2}=\sigma^{2}$ for all $g$. Thus, $h_{G}(t) Z_{\text {pool }}^{t}$ is also inadmissible for $\left(\sigma_{g}^{2}\right)^{t}$ unless $\sigma_{g}^{2}=\sigma^{2}$ for all $g$.

In Appendix 2 we prove that

Theorem 2. For any fixed $G$ and non-zero $t>-\nu / 2$, as $\nu \rightarrow \infty$,

(i) $\alpha_{1}^{*} \rightarrow 0$ when $\sigma_{g}^{2}$ are not all the same;

(ii) $R_{1}\left(\boldsymbol{\sigma}^{2 t}, \hat{\boldsymbol{\sigma}}^{2 t}\right)$ approaches to a constant function of $\alpha$ when $\sigma_{g}^{2}=\sigma^{2}$ for all $g$. 
Theorem 2 indicates that it is unnecessary to borrow information from other genes when the number of replications for each gene is large.

\subsection{Optimal Estimator under the Squared Loss Function}

Under the squared loss function (5), it is easy to check that the average risk is

$$
\begin{aligned}
R_{2}\left(\boldsymbol{\sigma}^{2 t}, \hat{\boldsymbol{\sigma}}^{2 t}\right) \triangleq & \frac{1}{G} \sum_{g=1}^{G} \mathrm{E}\left(L_{2}\left(\sigma_{g}^{2 t}, \hat{\sigma}_{g}^{2 t}\right)\right) \\
= & \frac{h_{G}^{2 \alpha}(t) h_{1}^{2(1-\alpha)}(t)}{h_{1}^{G-1}\left(\frac{2 \alpha t}{G}\right) h_{1}\left(2\left(1-\alpha+\frac{\alpha}{G}\right) t\right)}\left(\sigma_{\text {pool }}^{2}\right)^{2 \alpha t} \frac{1}{G} \sum_{g=1}^{G}\left(\sigma_{g}^{2}\right)^{-2 \alpha t} \\
& -\frac{2 h_{G}^{\alpha}(t) h_{1}^{1-\alpha}(t)}{h_{1}^{G-1}\left(\frac{\alpha t}{G}\right) h_{1}\left(\left(1-\alpha+\frac{\alpha}{G}\right) t\right)}\left(\sigma_{\text {pool }}^{2}\right)^{\alpha t} \frac{1}{G} \sum_{g=1}^{G}\left(\sigma_{g}^{2}\right)^{-\alpha t}+1,
\end{aligned}
$$

where $t>-\nu / 4$. We prove in Appendix 3 that

Theorem 3. For any fixed $G, \nu$ and non-zero power $t>-\nu / 4$, we have

(i) $\left.R_{2}^{\prime}\left(\boldsymbol{\sigma}^{2 t}, \hat{\boldsymbol{\sigma}}^{2 t}\right)\right|_{\alpha=0}<0$;

(ii) $\left.R_{2}^{\prime}\left(\boldsymbol{\sigma}^{2 t}, \hat{\boldsymbol{\sigma}}^{2 t}\right)\right|_{\alpha=1}>0$.

Theorem 3 implies that the gene-specific estimator, $h_{1}(t) Z_{g}^{t}$, is inadmissible. Contrary to the result under Stein loss function, the pooled estimator, $h_{G}(t) Z_{\text {pool }}^{t}$, is also inadmissible even when $\sigma_{g}^{2}=\sigma^{2}$ for all $g$. By Theorem 3 and the fact that $R_{2}\left(\boldsymbol{\sigma}^{2 t}, \hat{\boldsymbol{\sigma}}^{2 t}\right) \geq 0$, there exists an $\alpha_{2}^{*}$ that minimizes $R_{2}\left(\boldsymbol{\sigma}^{2 t}, \hat{\boldsymbol{\sigma}}^{2 t}\right)$. However, $R_{2}\left(\boldsymbol{\sigma}^{2 t}, \hat{\boldsymbol{\sigma}}^{2 t}\right)$ is not guaranteed to be a convex function of $\alpha$ in $[0,1]$. Therefore, $\alpha_{2}^{*}$ may not be unique. A counter example with very large $\nu$ is provided in Appendix 4. Nevertheless, for small $\nu, R_{2}\left(\boldsymbol{\sigma}^{2 t}, \hat{\boldsymbol{\sigma}}^{2 t}\right)$ is always strictly convex in millions of simulations under various situations. Denote the optimal estimator under the squared loss function as $\hat{\sigma}_{g}^{2 t}\left(\alpha_{2}^{*}\right)$. In Appendix 4 we also prove that

Theorem 4. For any fixed $G$ and non-zero $t>-\nu / 4$, as $\nu \rightarrow \infty$,

(i) $\alpha_{2}^{*} \rightarrow 0$ when $\sigma_{g}^{2}$ are not all the same;

(ii) $R_{2}\left(\boldsymbol{\sigma}^{2 t}, \hat{\boldsymbol{\sigma}}^{2 t}\right)$ approaches to a constant function of $\alpha$ when $\sigma_{g}^{2}=\sigma^{2}$ for all $g$.

\subsection{Estimation of the Optimal Shrinkage Parameters}

Both $\alpha_{1}^{*}$ and $\alpha_{2}^{*}$ depend on the unknown quantity

$$
b\left(\boldsymbol{\sigma}^{2}, \eta\right)=\left(\sigma_{\text {pool }}^{2}\right)^{\eta} \frac{1}{G} \sum_{g=1}^{G}\left(\sigma_{g}^{2}\right)^{-\eta},
$$

where $\eta=\alpha t$ or $\eta=2 \alpha t$. A simple estimator of $b\left(\boldsymbol{\sigma}^{2}, \eta\right)$ is $b(\mathbf{Z}, \eta)$, where $\mathbf{Z}=\left(Z_{1}, \ldots, Z_{G}\right)$. Denote $\hat{\alpha}_{1}^{*}$ and $\hat{\alpha}_{2}^{*}$ as the estimates of $\alpha_{1}^{*}$ and $\alpha_{2}^{*}$ with $b\left(\boldsymbol{\sigma}^{2}, \eta\right)$ in $(8)$ and $(9)$ replaced by $b(\mathbf{Z}, \eta)$. The following theorem shows that $\sigma_{g}^{2 t}\left(\hat{\alpha}_{1}^{*}\right)$ and $\sigma_{g}^{2 t}\left(\hat{\alpha}_{2}^{*}\right)$ are asymptotically optimal, and $\hat{\alpha}_{1}^{*}$ and $\hat{\alpha}_{2}^{*}$ are consistent under certain conditions as $\nu \rightarrow \infty$.

Theorem 5. Assume that $Z_{g} \stackrel{\text { a.s. }}{\rightarrow} \sigma_{g}^{2}$ as $\nu \rightarrow \infty, g=1, \ldots, G$. For any fixed $G$ and non-zero $t$, when $\nu \rightarrow \infty$, we have 
(i) $b(\mathbf{Z}, \alpha t) \stackrel{\text { a.s. }}{\rightarrow} b\left(\boldsymbol{\sigma}^{2}, \alpha t\right)$ uniformly for $\alpha \in[0,1]$;

(ii) $R_{k}\left(\boldsymbol{\sigma}^{2 t}, \hat{\boldsymbol{\sigma}}^{2 t}\left(\hat{\alpha}_{k}^{*}(\nu)\right)\right)-R_{k}\left(\boldsymbol{\sigma}^{2 t}, \hat{\boldsymbol{\sigma}}^{2 t}\left(\alpha_{k}^{*}(\nu)\right)\right) \stackrel{\text { a.s. }}{\rightarrow} 0, k=1,2$;

(iii) $\hat{\alpha}_{1}^{*}(\nu) \stackrel{\text { a.s. }}{\rightarrow} 0$ and $\hat{\alpha}_{2}^{*}(\nu) \stackrel{\text { a.s. }}{\rightarrow} 0$ when $\sigma_{g}^{2}$ are not all the same.

The proof of Theorem 5 is in Appendix 5. For microarray data, $\nu$ is small and $G$ is large. In the following we investigate asymptotic properties as $G \rightarrow \infty$. We now consider $\sigma_{g}^{2}$ as random variables and assume that $\sigma_{g}^{2} \stackrel{i i d}{\sim} F, g=1, \ldots, G$. We prove in Appendix 6 that

Lemma 2. For any fixed non-zero $t$ with $\nu>2 t, \mathrm{E}\left(\sigma_{1}^{2}\right)^{-t}<\infty$ and $\mathrm{E}\left(\ln \left(\sigma_{1}^{2}\right)\right)<\infty$, we have $w(\alpha t) b(\mathbf{Z}, \alpha t)-b\left(\boldsymbol{\sigma}^{2}, \alpha t\right) \stackrel{\text { a.s. }}{\rightarrow} 0$ uniformly for $\alpha \in[0,1]$ as $G \rightarrow \infty$, where $w(\alpha t)=$ $(\nu / 2)^{\alpha t} h_{1}(-\alpha t) \exp [-\alpha t \Psi(\nu / 2)]$.

For a fixed $t$, let $H_{k}\left(\boldsymbol{\sigma}^{2}, \alpha, G\right)=R_{k}\left(\boldsymbol{\sigma}^{2 t}, \hat{\boldsymbol{\sigma}}^{2 t}(\alpha)\right), H_{k}(\mathbf{Z}, \alpha, G)$ be the functions with $b\left(\boldsymbol{\sigma}^{2}, k \alpha t\right)$ in $H_{k}\left(\boldsymbol{\sigma}^{2}, \alpha, G\right)$ replaced by $w(k \alpha t) b(\mathbf{Z}, k \alpha t), k=1,2$. Denote $\alpha_{k}^{*}(G)=\underset{\alpha \in[0,1]}{\operatorname{argmin}} H_{k}\left(\boldsymbol{\sigma}^{2}, \alpha, G\right)$ and $\breve{\alpha}_{k}^{*}(G)=\underset{\alpha \in[0,1]}{\operatorname{argmin}} H_{k}(\mathbf{Z}, \alpha, G)$.

Theorem 6. For any fixed non-zero $t$,

(i) when $\nu>2|t|, \mathrm{E}\left(\sigma_{1}^{2}\right)^{-t}<\infty$ and $\mathrm{E}\left(\ln \left(\sigma_{1}^{2}\right)\right)<\infty$, we have $R_{1}\left(\boldsymbol{\sigma}^{2 t}, \hat{\boldsymbol{\sigma}}^{2 t}\left(\breve{\alpha}_{1}^{*}(G)\right)\right)-$ $R_{1}\left(\boldsymbol{\sigma}^{2 t}, \hat{\boldsymbol{\sigma}}^{2 t}\left(\alpha_{1}^{*}(G)\right)\right) \stackrel{\text { a.s. }}{\rightarrow} 0$ and $\breve{\alpha}_{1}^{*}(G)-\alpha_{1}^{*}(G) \stackrel{\text { a.s. }}{\rightarrow} 0$ as $G \rightarrow \infty$;

(ii) when $\nu>4|t|, \mathrm{E}\left(\sigma_{1}^{2}\right)^{-2 t}<\infty$ and $\mathrm{E}\left(\ln \left(\sigma_{1}^{2}\right)\right)<\infty$, we have $R_{2}\left(\boldsymbol{\sigma}^{2 t}, \hat{\boldsymbol{\sigma}}^{2 t}\left(\breve{\alpha}_{2}^{*}(G)\right)\right)-$ $R_{2}\left(\boldsymbol{\sigma}^{2 t}, \hat{\boldsymbol{\sigma}}^{2 t}\left(\alpha_{2}^{*}(G)\right)\right) \stackrel{\text { a.s. }}{\rightarrow} 0$ as $G \rightarrow \infty$.

The proof of Theorem 6 is in Appendix 6. Note that there is no corresponding consistent result for $\alpha_{2}^{*}$ since it may not be unique. For the special case that $F$ is a Gamma distribution with shape parameter $\gamma$ and scale parameter $\beta$, it is easy to check that $E\left(\ln \left(\sigma_{1}^{2}\right)\right)=\Psi(\gamma)+\ln \beta<$ $\infty, \mathrm{E}\left(\sigma_{1}^{2}\right)^{-t}=\beta^{-t} \Gamma(\gamma-t) / \Gamma(\gamma)<\infty$ for $\gamma>t$, and $\mathrm{E}\left(\sigma_{1}^{2}\right)^{-2 t}=\beta^{-2 t} \Gamma(\gamma-2 t) / \Gamma(\gamma)<\infty$ for $\gamma>2 t$.

Note that Theorem 6 does not apply for small $\nu$. We propose an alternative two-step procedure: (i) substitute $b\left(\boldsymbol{\sigma}^{2}, \eta\right)$ in $(8)$ and $(9)$ by $b(\mathbf{Z}, \eta)$, and compute temporary optimal shrinkage parameters and the corresponding shrinkage estimators, say $\hat{\boldsymbol{\sigma}}_{-}^{2}$; (ii) substitute $b\left(\boldsymbol{\sigma}^{2}, \eta\right)$ in $(8)$ and $(9)$ by $b\left(\hat{\boldsymbol{\sigma}}_{-}^{2}, \eta\right)$ to get the final optimal shrinkage parameters $\check{\alpha}_{1}^{*}$ and $\check{\alpha}_{2}^{*}$. When $t>0$, since $\sigma_{g}^{2}$ appears in the denominator in (8) and (9), extreme small values of $Z_{g}$ make estimates of $\alpha_{1}^{*}$ and $\alpha_{2}^{*}$ unstable. We truncate the smallest $1 \%$ of $Z_{g}$ 's in our procedures. We find that the truncation is unnecessary when $t<0$. Simulations indicate that $\check{\alpha}_{1}^{*}$ and $\check{\alpha}_{2}^{*}$ perform better than $\hat{\alpha}_{1}^{*}, \hat{\alpha}_{2}^{*}, \breve{\alpha}_{1}^{*}$ and $\breve{\alpha}_{2}^{*}$ when $\nu$ is small. Therefore, $\check{\alpha}_{1}^{*}$ and $\check{\alpha}_{2}^{*}$ will be used in our simulations.

\section{SIMULATIONS AND COMPARISONS}

In this section we conduct simulations to compare the performance of our estimators with the CHQBC estimator and the modified CHQBC estimator for the purpose of estimation. All estimators considered in this section perform substantially better than the standard genespecific estimator. For simplicity, we will not present the results for the gene-specific estimator. We evaluate the performance for estimating $\sigma_{g}^{2}$ in Section 4.1 and the performance for estimating $\left(\sigma_{g}^{2}\right)^{-1}$ in Section 4.2. We set $G=5000$ in this section.

\subsection{Estimation of $\sigma_{g}^{2}$}


We consider four different estimators in this subsection: $\hat{\sigma}_{g}^{2}\left(\check{\alpha}_{1}^{*}\right), \hat{\sigma}_{g}^{2}\left(\check{\alpha}_{2}^{*}\right), \hat{\sigma}_{g}^{2}\left(\hat{\alpha}_{0}\right)$ and $\tilde{\sigma}_{g}^{2}\left(\hat{\alpha}_{0}\right)$. We simulate $\sigma_{g}^{2}, g=1, \ldots, G$, from a Gamma distribution with shape parameter $\gamma$ and scale parameter $\beta$. To evaluate performance under different levels of variance heterogeneity, we consider three different shape parameters, $\gamma=0.25,1$ and 4 , which corresponds to three different coefficients of variation $\left(C V=\sqrt{\gamma \beta^{2}} /(\gamma \beta)=\sqrt{\gamma} / \gamma\right)$ at levels 2,1 and 0.5 respectively. For each $\sigma_{g}^{2}$, we simulate $\nu+1$ observations from $N\left(\mu_{g}, \sigma_{g}^{2}\right)$, where $\mu_{g}$ is a random sample from $N(0,1)$. We calculate $Z_{g}$ as the sample variance for each $g$. We use a factorial design which consists 3 levels for $\gamma$ and 7 levels for $\nu, \nu=1, \ldots, 7$. Therefore, we have 21 combinations of parameter settings. For each setting, we repeat simulation 100 times. We compute the average risk

$$
A R_{k}=\frac{1}{100 G} \sum_{r=1}^{100} \sum_{g=1}^{G} L_{k}\left(\sigma_{g r}^{2}, \hat{\sigma}_{g r}^{2}\right), \quad k=1,2,
$$

where $r$ represents simulation replications, and $k=1$ and $k=2$ correspond to Stein and the squared loss functions respectively. We plot $\ln \left(A R_{k}\right)$ as a function of $\nu$ for all four methods in Figure 1.

The modified CHQBC estimator $\hat{\sigma}_{g}^{2}\left(\hat{\alpha}_{0}\right)$ has smaller average risk than the original CHQBC estimator $\tilde{\sigma}_{g}^{2}\left(\hat{\alpha}_{0}\right)$ in all settings. When the variance heterogeneity is not small $(\gamma=0.25$ and $\gamma=1)$, two optimal estimators $\hat{\sigma}_{g}^{2}\left(\check{\alpha}_{1}^{*}\right)$ and $\hat{\sigma}_{g}^{2}\left(\check{\alpha}_{2}^{*}\right)$ have similar risks which are smaller than those of $\hat{\sigma}_{g}^{2}\left(\hat{\alpha}_{0}\right)$. When $\nu$ and the variance heterogeneity is small $(\gamma=4), \hat{\sigma}_{g}^{2}\left(\hat{\alpha}_{0}\right)$ has smaller risks under Stein loss function than the optimal estimators. Overall, the optimal estimator under Stein loss function performs well. Note that the estimates of optimal shrinkage parameters $\check{\alpha}_{1}^{*}$ and $\check{\alpha}_{2}^{*}$ do not guarantee the optimal performance, especially when $\nu$ is small. Simulations (not shown) indicate that $\alpha_{1}^{*}$ and $\alpha_{2}^{*}$ do guarantee the optimal performance for Stein and the squared loss functions respectively.

To take a closer look at estimates of shrinkage parameters, we construct boxplots of ratios, $\check{\alpha}_{1}^{*} / \hat{\alpha}_{0}$ and $\check{\alpha}_{2}^{*} / \hat{\alpha}_{0}$ for $\gamma=1$ in Figure 2. Boxplots of ratios for $\gamma=0.25$ and $\gamma=4$ are similar (not shown). In general, $\check{\alpha}_{1}^{*}>\check{\alpha}_{2}^{*}, \check{\alpha}_{1}^{*}<\hat{\alpha}_{0}$ and $\check{\alpha}_{2}^{*}<\hat{\alpha}_{0}$ for small $\nu$, and $\check{\alpha}_{1}^{*}>\hat{\alpha}_{0}$ and $\check{\alpha}_{2}^{*}>\hat{\alpha}_{0}$ for large $\nu$. Therefore, $\hat{\sigma}_{g}^{2}\left(\check{\alpha}_{1}^{*}\right)$ shrinks more than $\hat{\sigma}_{g}^{2}\left(\check{\alpha}_{2}^{*}\right)$, and both $\hat{\sigma}_{g}^{2}\left(\check{\alpha}_{1}^{*}\right)$ and $\hat{\sigma}_{g}^{2}\left(\check{\alpha}_{2}^{*}\right)$ shrink less than $\hat{\sigma}_{g}^{2}\left(\hat{\alpha}_{0}\right)$ and $\tilde{\sigma}_{g}^{2}\left(\hat{\alpha}_{0}\right)$ when $\nu$ is small. This explains why the optimal shrinkage estimators are inferior to $\hat{\sigma}_{g}^{2}\left(\hat{\alpha}_{0}\right)$ when the variance heterogeneity and $\nu$ are both small.

\subsection{Estimation of $\sigma_{g}^{-2}$}

As discussed in Section 1, it is better to use an estimator of $\sigma_{g}^{-2}$ directly than the reciprocal of an estimator of $\sigma_{g}^{2}$ in the $F$-test (Section 5). In this subsection we evaluate performance for estimating $\sigma_{g}^{-2}$.

Simulations (not shown) indicate that $\hat{\sigma}_{g}^{-2}\left(\check{\alpha}_{1}^{*}\right)$ always performs better than $\hat{\sigma}_{g}^{-2}\left(\check{\alpha}_{2}^{*}\right)$. For simplicity, we present results for $\hat{\sigma}_{g}^{-2}\left(\check{\alpha}_{1}^{*}\right)$ only. We consider four estimators: $\hat{\sigma}_{g}^{-2}\left(\check{\alpha}_{1}^{*}\right), \hat{\sigma}_{g}^{2}\left(\check{\alpha}_{1}^{*}\right)$, $\hat{\sigma}_{g}^{2}\left(\hat{\alpha}_{0}\right)$ and $\tilde{\sigma}_{g}^{2}\left(\hat{\alpha}_{0}\right)$, where $\hat{\sigma}_{g}^{-2}\left(\check{\alpha}_{1}^{*}\right)$ is the estimator of $\left(\sigma_{g}^{2}\right)^{t}$ with $t=-1$. We take the reciprocal of the last three as estimators of $\sigma_{g}^{-2}$. Theorem 1 requires that $\nu \geq 3$ when $t=-1$. Therefore, 

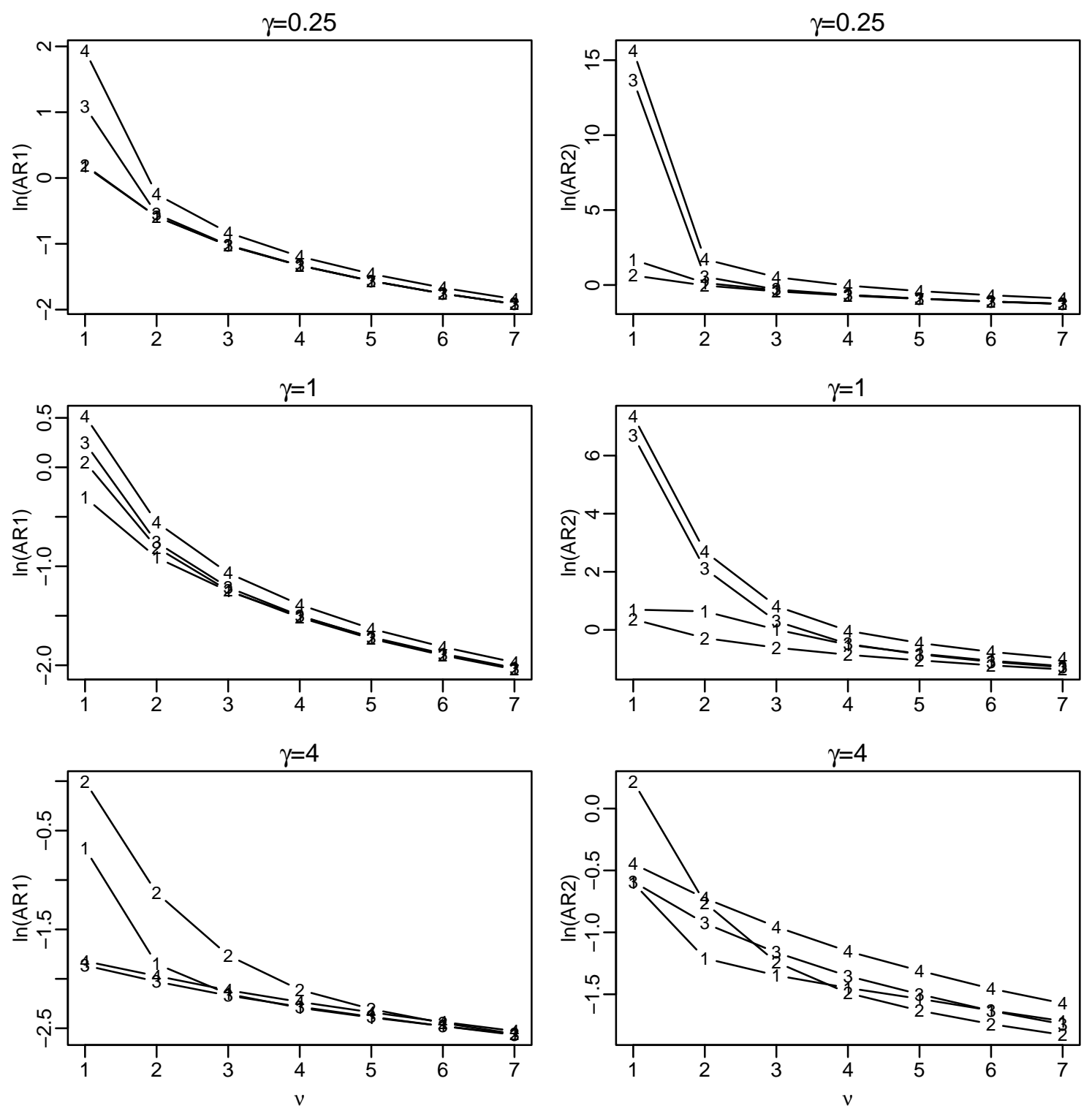

Figure 1: Plots of average risk for estimating $\sigma_{g}^{2}$ under Stein loss function (left) and the squared loss function (right). Three rows correspond to three shape parameters. Four lines in each plot marked "1", "2", "3" and "4" correspond to the optimal estimator under Stein loss function $\hat{\sigma}_{g}^{2}\left(\check{\alpha}_{1}^{*}\right)$, the optimal estimator under the squared loss function $\hat{\sigma}_{g}^{2}\left(\check{\alpha}_{2}^{*}\right)$, the modified CHQBC estimator $\hat{\sigma}_{g}^{2}\left(\hat{\alpha}_{0}\right)$ and the CHQBC estimator $\tilde{\sigma}_{g}^{2}\left(\hat{\alpha}_{0}\right)$ respectively. 

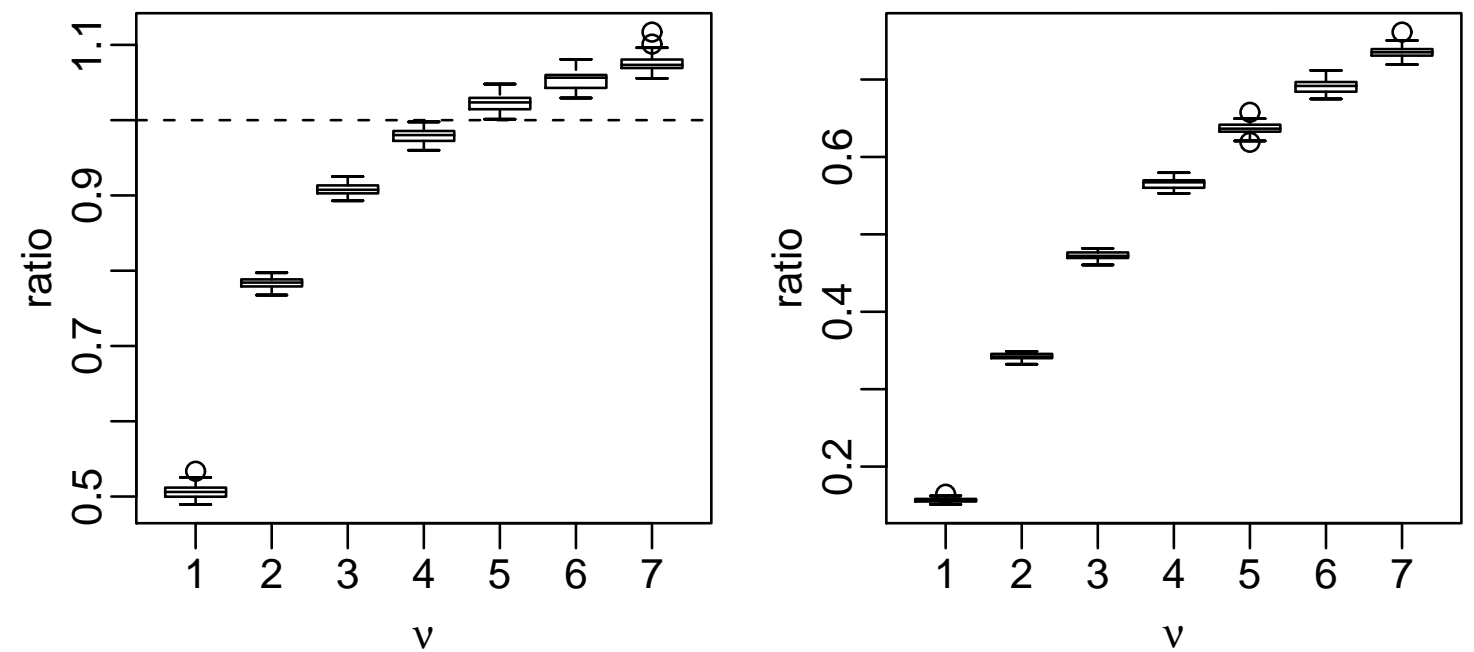

Figure 2: Boxplots of ratio $=\check{\alpha}_{1}^{*} / \hat{\alpha}_{0}$ (left) and ratio $=\check{\alpha}_{2}^{*} / \hat{\alpha}_{0}$ (right) with $\gamma=1$.

we take $\nu$ from 3 to 9 . All other settings are the same as those in Section 4.1.

Figure 2 shows that under both Stein and the squared loss functions, risk of $\hat{\sigma}_{g}^{-2}\left(\check{\alpha}_{1}^{*}\right)<$ risk of $\tilde{\sigma}_{g}^{2}\left(\hat{\alpha}_{0}\right)<$ risk of $\hat{\sigma}_{g}^{2}\left(\hat{\alpha}_{0}\right)<$ risk of $\hat{\sigma}_{g}^{2}\left(\check{\alpha}_{1}^{*}\right)$. It is interesting to note that $\tilde{\sigma}_{g}^{2}\left(\hat{\alpha}_{0}\right)$ outperforms $\hat{\sigma}_{g}^{2}\left(\hat{\alpha}_{0}\right)$, which, again, confirms that a better estimator for $\sigma_{g}^{2}$ may not lead to a better estimator for $\sigma_{g}^{-2}$. We have performed many more simulations with different parameters for both Sections 4.1 and 4.2. Comparative results remain the same.

\section{APPLICATIONS}

Cui et al. (2005) has demonstrated that the F-test using the CHQBC estimator has the best or nearly the best power among several "information-sharing" statistics for detecting differentially expressed genes over a wide range of settings. For simplicity, we compare the performance of our shrinkage estimators with the CHQBC estimator and the modified CHQBC estimator only. We introduce $F$-like statistics using shrinkage estimators in Section 5.1. In Section 5.2, we apply our method to a microarray data and conduct simulations to evaluate and compare the performances of several $F$-like statistics.

\section{$5.1 F$-like statistics}

For a fixed gene $g, g=1, \ldots, G$, test statistics are usually based on the following general linear mixed effects model (Kerr, Afshari, Bennett, Bushel, Martinez, Walker and Churchill 2002, Cui et al. 2005)

$$
\mathbf{y}_{g}=\mathbf{X}_{g} \boldsymbol{\beta}_{g}+\mathbf{Z}_{g} \mathbf{b}_{g}+\boldsymbol{\epsilon}_{g}
$$

where $\mathbf{y}_{g}$ is the vector of all observations for gene $g, \mathbf{X}_{g}$ and $\mathbf{Z}_{g}$ are design matrices for the fixed effects $\boldsymbol{\beta}_{g}$ and the random effects $\mathbf{b}_{g}$ respectively, and $\boldsymbol{\epsilon}_{g}$ is the vector of random errors. 

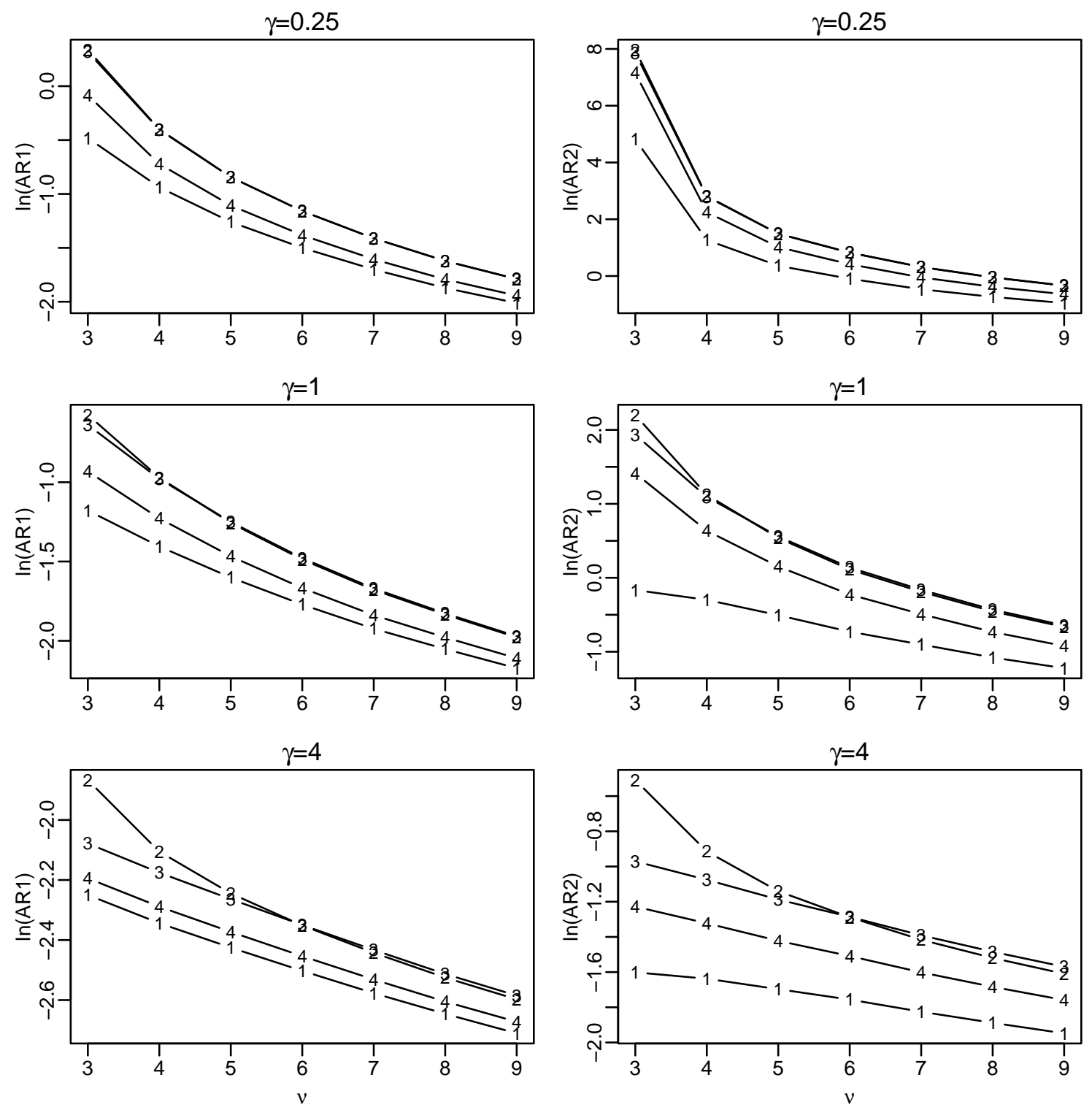

Figure 3: Plots of average risk for estimating $\left(\sigma_{g}^{2}\right)^{-1}$ under Stein loss function (left) and the squared loss function (right). Three rows correspond to three shape parameters. Four lines in each plot marked "1", "2", "3" and "4" correspond to $\hat{\sigma}_{g}^{-2}\left(\check{\alpha}_{1}^{*}\right), \hat{\sigma}_{g}^{2}\left(\check{\alpha}_{1}^{*}\right), \hat{\sigma}_{g}^{2}\left(\hat{\alpha}_{0}\right)$ and $\tilde{\sigma}_{g}^{2}\left(\hat{\alpha}_{0}\right)$. 
We assume that

$$
\left(\begin{array}{c}
\mathbf{b}_{g} \\
\boldsymbol{\epsilon}_{g}
\end{array}\right) \sim N\left(\left(\begin{array}{l}
\mathbf{0} \\
\mathbf{0}
\end{array}\right), \sigma_{g}^{2}\left(\begin{array}{ll}
\mathbf{G}_{g} & \\
& \mathbf{R}_{g}
\end{array}\right)\right),
$$

where $\sigma_{g}^{2}$ is the error variance for gene $g$. Note that the general linear model is a special case with empty $\mathbf{Z}_{g}$ and $\mathbf{b}_{g}$. Denote $\hat{\boldsymbol{\beta}}_{g}$ and $\hat{\mathbf{b}}_{g}$ as the best linear unbiased predictor and its variance-covariance matrix

$$
\mathbf{C}_{g}=\sigma_{g}^{2}\left(\begin{array}{cc}
\mathbf{X}_{g}^{\prime} \mathbf{R}_{g}^{-1} \mathbf{X}_{g} & \mathbf{X}_{g}^{\prime} \mathbf{R}_{g}^{-1} \mathbf{Z}_{g} \\
\mathbf{Z}_{g}^{\prime} \mathbf{R}_{g}^{-1} \mathbf{X}_{g} & \mathbf{Z}_{g}^{\prime} \mathbf{R}_{g}^{-1} \mathbf{Z}_{g}+\mathbf{G}_{g}^{-1}
\end{array}\right)^{-} \triangleq \sigma_{g}^{2} \mathbf{D}_{g},
$$

where " -" sign represents the generalized inverse of the matrix. The $F$ statistic for testing the hypothesis $H_{0}: \mathbf{L}_{g}^{\prime} \boldsymbol{\beta}_{g}=0$ is (Littell, Milliken, Stroup and Wolfinger 1996)

$$
F=\frac{\hat{\boldsymbol{\beta}}_{g}^{\prime} \mathbf{L}_{g}\left(\mathbf{L}_{g}^{\prime} \hat{\mathbf{D}}_{g} \mathbf{L}_{g}\right)^{-1} \mathbf{L}_{g}^{\prime} \hat{\boldsymbol{\beta}}_{g} / \operatorname{rank}\left(\mathbf{L}_{g}\right)}{\hat{\sigma}_{g}^{2}} \triangleq \Delta_{g} \hat{\sigma}_{g}^{-2},
$$

where $\hat{\mathbf{D}}_{g}$ is an estimator of $\mathbf{D}_{g}$ which can be calculated using the restricted maximum likelihood method (Searle, Casella and McCulloch 1992), and $\hat{\sigma}_{g}^{-2}$ is an estimator of $\sigma_{g}^{-2}$.

Different estimators of $\sigma_{g}^{-2}$ leads to different $F$-like statistics. The gene-specific $F$ statistic replaces $\sigma_{g}^{2}$ by its gene-specific estimator. We will consider four $F$-like statistics, $F_{1}, F_{2}, F_{3}$ and $F_{4}$, with $\hat{\sigma}_{g}^{-2}$ in (12) replaced by $\hat{\sigma}_{g}^{-2}\left(\check{\alpha}_{1}^{*}\right), \hat{\sigma}_{g}^{-2}\left(\check{\alpha}_{2}^{*}\right), 1 / \hat{\sigma}_{g}^{2}\left(\hat{\alpha}_{0}\right)$ and $1 / \tilde{\sigma}_{g}^{2}\left(\hat{\alpha}_{0}\right)$ respectively. $F_{4}$ is the same as the $F_{s}$ in Cui et al. (2005). Since $F_{4}\left(F_{s}\right)$ compares favorably to other $F$-like statistics including the gene-specific $F$-statistic (Cui et al. 2005), we will compare our methods to $F_{4}\left(F_{s}\right)$ only. Simulations (not shown) indicate that $F$-like statistics with $\hat{\sigma}_{g}^{2}$ in (12) replaced by $\hat{\sigma}_{g}^{2}\left(\check{\alpha}_{1}^{*}\right)$ or $\hat{\sigma}_{g}^{2}\left(\check{\alpha}_{2}^{*}\right)$ have similar performance as $F_{3}$. To save space, these results are not presented. Since $F$-like statistics do not follow $F$ distributions, we calculate $p$-values by permutation as in Cui et al. (2005). We note that the method described here applies to general designs, and usually $\mathbf{X}_{g}, \mathbf{Z}_{g}, \mathbf{G}_{g}, \mathbf{R}_{g}$ and $\mathbf{L}_{g}$ are independent of $g$.

\subsection{Case study}

Cui et al. (2005) described an experiment that compared two human colon cancer cell lines, CACO2 and HCT116, and three human ovarian cancer cell lines, ES2, MDAH2774 and OV1063. Five samples were arranged in a loop and no reference sample was used. Fluorescent dye labeled cDNA targets were hybridized to cDNA microarrays containing 9600 human cDNA clones. To simplify the analysis, as in Cui et al. (2005), the duplicated spots for the same gene on each array were averaged at the original signal level. Observations were transformed and normalized.

We fit the following model to each gene (Cui et al. 2005):

$$
y_{i j}=\mu+A_{i}+D_{j}+S_{k(i, j)}+\epsilon_{i j}, \quad i=1, \ldots, 10 ; j=1,2 ; k=1, \ldots, 5,
$$

where $\mu$ is the gene mean, $A_{i}$ is the array effect, $D_{j}$ is the dye effect, $S_{k(i, j)}$ is the sample effect, and $\epsilon_{i j}$ is the random error. In general, the terms $\mu, D_{j}$ and $S_{k(i, j)}$ are fixed effects and $A_{i}$ 
are random effects. Cui et al. (2005) demonstrated that the array variance has little impact on the $F$-tests. Therefore, as Cui et al. (2005), we treat $A_{i}$ as fixed effects. All analyses are conducted using the newest version of R/MAANOVA (Wu and Churchill 2005). At a nominal significant level of $0.01, F_{1}, F_{2}, F_{3}$ and $F_{4}$ detected 1877, 1870, 1790 and 1838 significant genes respectively.

To study the false positive and successful detection rates for four $F$-like tests, we simulate 100 data sets using the same design as the real data. Each simulated data set contains 1000 constant genes and 1000 differentially expressed genes. Since the successful detection rate of a test depends on the magnitude of the overall treatment effect, $\Theta=\sum_{k=1}^{5}\left(S_{k}-\bar{S} \text {. }\right)^{2} / 4$, where $S_{k}$ is defined in (13), we consider $\sqrt{4 \Theta}$ as a parameter. Specifically, we generate $S_{k}=\sqrt{4 \Theta}\left(Q_{k}-\bar{Q}\right) / \sqrt{\sum_{k=1}^{5}\left(Q_{k}-\bar{Q}\right)^{2}}$ where $Q_{k} \stackrel{i i d}{\sim} N(0,1)$, with $\sqrt{4 \Theta}=0.1,0.2, \ldots, 1$ representing ten different levels of treatment effects. The fixed effects $\mu$ and $D_{j}$ are drawn randomly from the normal distributions $N\left(0,0.65^{2}\right)$ and $N\left(0,0.35^{2}\right)$ respectively, and are held constant across all simulations. For each simulation, $A_{i}$ and $\epsilon_{i j}$ are drawn randomly from $N\left(0,0.6^{2}\right)$ and $N\left(0, \sigma_{g}^{2}\right)$ respectively, where $\sigma_{g}^{2}$ are sampled randomly without replacement from the 9600 estimates of residual variances of the real data. As in Cui et al. (2005), the variability of the residual variances was controlled by a parameter $\tau$ through the formula $\sigma_{g, \tau}^{2}=\left(\sigma_{g}^{2 \tau} / \sigma_{\text {pool }}^{2 \tau}\right) \sigma_{\text {pool }}^{2}$. We consider three choices of $\tau, \tau=0.5,1$ and 2 , which correspond to $C V=0.63,1.84$ and 10.60 respectively.

\begin{tabular}{|c|c|c|c|c|}
\hline & $F_{1}$ & $F_{2}$ & $F_{3}$ & $F_{4}$ \\
\hline$C V=0.63$ & 0.026 & 0.027 & 0.024 & 0.026 \\
\hline$C V=1.84$ & 0.038 & 0.038 & 0.041 & 0.041 \\
\hline$C V=10.6$ & 0.046 & 0.045 & 0.048 & 0.047 \\
\hline
\end{tabular}

Table 1: Average false positive rates of four $F$-like statistics at the significance level 0.05 .

Average false positive rates at the significance level 0.05 listed in Table 1 indicate that the type I error is under the nominal value. All four $F$-like statistics have similar false positive rates. $F_{3}$ and $F_{4}$ have similar powers in all settings even though the performances of $\hat{\sigma}_{g}^{2}\left(\hat{\alpha}_{0}\right)$ and $\tilde{\sigma}_{g}^{2}\left(\hat{\alpha}_{0}\right)$ are quite different for estimation (Section 4$)$. Improvements of the new $F$-like tests come with moderate to large treatment effect. For simplicity, in Figure 4, we plot the successful detection rate for $F_{1}, F_{2}$ and $F_{4}$ with $0.4 \leq \sqrt{4 \Theta} \leq 1$. $F_{1}$ outperforms $F_{4}$ in all situations. $F_{1}$ performs better than $F_{2}$ when the heterogeneity of variance is not large $(C V=0.63$ and $C V=1.84)$. When the heterogeneity of variance is large $(C V=10.6), F_{2}$ performs better than $F_{1}$. For microarray data in their typical range of $C V$, we recommend $F_{1}$.

\section{DISCUSSION}

One major challenge in microarray data analysis is the relative small number of replications for each gene compared to the large number of genes. In this paper we propose a family of 

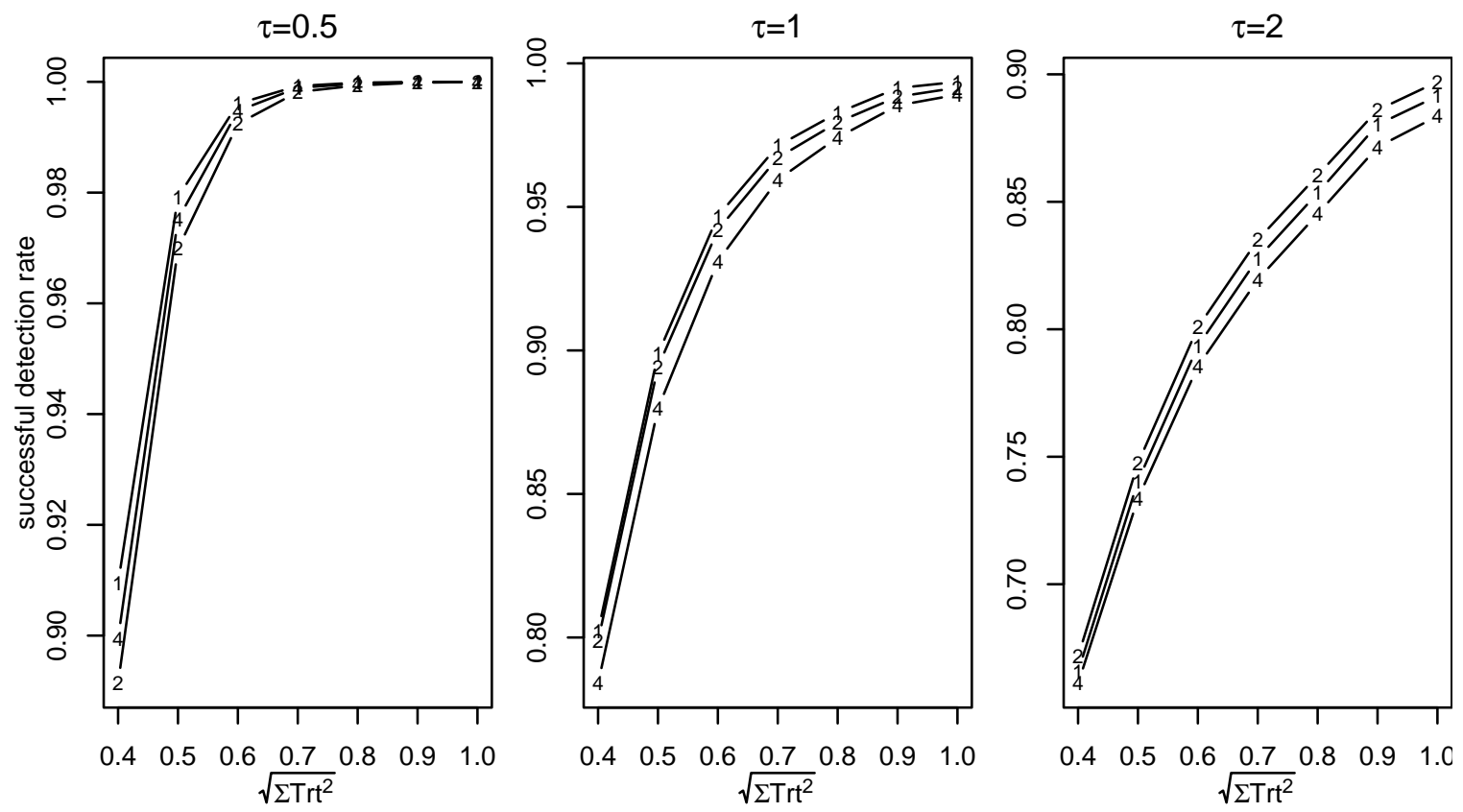

Figure 4: Plots of the average successful detection rate of three $F$-like statistics.

shrinkage variance estimators which borrow information across genes by shrinking each genespecific variance estimator toward the bias corrected geometric mean of variance estimators for all genes. The amount of optimal shrinkage depends on the variability of the individual variances. We have shown that the standard sample variance is inadmissible under either Stein or the squared loss functions. Our optimal shrinkage estimators compare favorably with the CHQBC estimator in terms of both estimation and hypothesis test. One key is to use an estimator of $\sigma_{g}^{-2}$ directly in the $F$ statistic instead of using the reciprocal of an estimator of $\sigma_{g}^{2}$. We note that the optimal shrinkage variance estimators may also be used for other purposes such as constructing confidence intervals. We recommend the estimator under Stein loss function for microarray data analysis.

On the logarithm scale, our shrinkage estimator (7) is a weighted average of the gene-specific variance and the bias corrected geometric mean. One of our future research topic is to consider a weighted average of the gene-specific variance and the arithmetic mean (Baldi and Long 2001). Our method shrinks all gene-specific variances to a unique common variance. One may also borrow information contained in the sample mean (Stein 1964). Better shrinkage estimator may be constructed when additional information is available. For example, instead of shrinking to the overall geometric mean, one may borrow information from neighboring genes (Baldi and Long 2001, Jain et al. 2003).

Even though motivated and applied to the microarray data, the optimal shrinkage variance estimators in this paper are general which can have a wide range of applications such as portfolio optimization (Ledoit and Wolf 2004a, Ledoit and Wolf 2004b). Our methodology and theory extend Stein's landmark results from shrinkage estimation of means to shrinkage estimation of 
variances (James and Stein 1961), and from shrinkage estimation of a single variance to the shrinkage estimation of multiple variances (Stein 1964).

\section{APPENDIX 1: THE PROOF OF THEOREM 1}

Lemma 3. (i) For any $t>0$,

$$
\begin{aligned}
\Psi(t+1) & =\Psi(t)+1 / t, \\
\Psi^{\prime}(t) & =\sum_{k=0}^{\infty}(t+k)^{-2}>0, \\
\Gamma^{\prime \prime}(t) / \Gamma(t) & =\Psi^{2}(t)+\Psi^{\prime}(t),
\end{aligned}
$$

where $\Psi(t)=\Gamma^{\prime}(t) / \Gamma(t)$ and $\Psi^{\prime}(t)$ are the digamma and trigamma functions;

(ii) For any $t>0, \Gamma(s) / \Gamma(s+t)$ is a strictly decreasing function of $s$ on $(0, \infty)$;

(iii) $h_{n}(t)>h_{1}(t)$ for any $n \geq 2$ and any non-zero $t>-\nu / 2$;

(iv) $h_{n}^{2}(t)>h_{1}^{n}(2 t / n)$ for any $n \geq 2$ and any non-zero $t>-\nu / 2$;

(v) $\lim _{\nu \rightarrow \infty} h_{n}(t)=1$ for any fixed $n$ and $t$.

[Proof] (i) (14) is the same as \#6.3.5 in Abramowitz and Stegun (1972). (15) is a special case of \#6.4.10 in Abramowitz and Stegun (1972) with $n=1$. (16) can be derived directly from the definition of $\Psi(t)$.

(ii) Let $B(s, t)=\int_{0}^{1} x^{s-1}(1-x)^{t-1} d x$ be the Beta function. It is obvious that $B(s, t)$ is a strictly decreasing function of $s$ for any fixed $t>0$. Therefore, $\Gamma(s) / \Gamma(s+t)=B(s, t) / \Gamma(t)$ is a strictly decreasing function of $s$.

(iii) Using Lemma 3 (ii), for any $t>0$,

$$
\begin{aligned}
h_{1}(t) & =\left(\frac{\nu}{2}\right)^{t} \frac{\Gamma\left(\frac{\nu}{2}\right)}{\Gamma\left(\frac{\nu}{2}+t\right)} \\
& =\left(\frac{\nu}{2}\right)^{t}\left(\frac{\Gamma\left(\frac{\nu}{2}\right)}{\Gamma\left(\frac{\nu}{2}+\frac{t}{n}\right)}\right)\left(\frac{\Gamma\left(\frac{\nu}{2}+\frac{t}{n}\right)}{\Gamma\left(\frac{\nu}{2}+\frac{2 t}{n}\right)}\right) \cdots\left(\frac{\Gamma\left(\frac{\nu}{2}+t-\frac{t}{n}\right)}{\Gamma\left(\frac{\nu}{2}+t\right)}\right) \\
& <\left(\frac{\nu}{2}\right)^{t}\left(\frac{\Gamma\left(\frac{\nu}{2}\right)}{\Gamma\left(\frac{\nu}{2}+\frac{t}{n}\right)}\right)\left(\frac{\Gamma\left(\frac{\nu}{2}\right)}{\Gamma\left(\frac{\nu}{2}+\frac{t}{n}\right)}\right) \cdots\left(\frac{\Gamma\left(\frac{\nu}{2}\right)}{\Gamma\left(\frac{\nu}{2}+\frac{t}{n}\right)}\right) \\
& =h_{n}(t) .
\end{aligned}
$$

Same result holds for $-\nu / 2<t<0$ by noting the fact that $\Gamma(s) / \Gamma(s+t)$ is a strictly increasing function of $s$ on $(-t, \infty)$ for $t<0$.

(iv) Similar arguments as in the proof of (iii) give

$$
\frac{h_{n}^{2}(t)}{h_{1}^{n}\left(\frac{2 t}{n}\right)}=\left(\frac{\Gamma\left(\frac{\nu}{2}\right)}{\Gamma\left(\frac{\nu}{2}+\frac{t}{n}\right)} / \frac{\Gamma\left(\frac{\nu}{2}+\frac{t}{n}\right)}{\Gamma\left(\frac{\nu}{2}+\frac{2 t}{n}\right)}\right)^{n}>1 .
$$

(v) $\lim _{\nu \rightarrow \infty} h_{n}(t)=1$ is an immediate result of the fact that $\lim _{t \rightarrow \infty} t^{b-a} \Gamma(t+a) / \Gamma(t+b)=1$ for any $a, b \in \mathcal{R}$ (\#6.1.46 in Abramowitz and Stegun (1972)). 
Lemma 4. For any positive sequence $\left\{a_{i}, i=1, \ldots, n\right\}$, we have

$$
\sum_{i=1}^{n} \frac{1}{a_{i}}\left(\frac{1}{n} \sum_{j=1}^{n} \ln \left(a_{j}\right)-\ln \left(a_{i}\right)\right) \geq 0 .
$$

[Proof] Without loss of generality, we assume that $0<a_{1} \leq \cdots \leq a_{n}$. Thus $1 / a_{1} \geq \cdots \geq 1 / a_{n}$. Let $b_{i}=\sum_{j=1}^{n} \ln \left(a_{j}\right) / n-\ln \left(a_{i}\right)$. Since that $b_{1} \geq \cdots \geq b_{n}$ and $\sum_{i=1}^{n} b_{i}=0$, there exists an $m \in\{1, \ldots, n\}$ such that $b_{1} \geq \cdots \geq b_{m} \geq 0 \geq b_{m+1} \geq \cdots \geq b_{n}$ and $\sum_{i=1}^{m} b_{i}=-\sum_{i=m+1}^{n} b_{i}$. Therefore,

$$
\begin{aligned}
\sum_{i=1}^{n} \frac{1}{a_{i}}\left(\frac{1}{n} \sum_{j=1}^{n} \ln \left(a_{j}\right)-\ln \left(a_{i}\right)\right) & =\sum_{i=1}^{m} \frac{1}{a_{i}} b_{i}+\sum_{i=m+1}^{n} \frac{1}{a_{i}} b_{i} \\
& \geq \frac{1}{a_{m}} \sum_{i=1}^{m} b_{i}+\frac{1}{a_{m+1}} \sum_{i=m+1}^{n} b_{i} \\
& =\left(\frac{1}{a_{m}}-\frac{1}{a_{m+1}}\right) \sum_{i=1}^{m} b_{i} \geq 0 .
\end{aligned}
$$

Proof of Theorem 1. For simplicity, we define

$$
A(\alpha)=C_{1}(\alpha) C_{2}(\alpha)-C_{3}(\alpha)
$$

where

$$
\begin{aligned}
& C_{1}(\alpha) \triangleq \frac{h_{G}^{\alpha}(t) h_{1}^{1-\alpha}(t)}{h_{1}^{G-1}\left(\frac{\alpha t}{G}\right) h_{1}\left(\left(1-\alpha+\frac{\alpha}{G}\right) t\right)} \\
& C_{2}(\alpha) \triangleq\left(\sigma_{\text {pool }}^{2}\right)^{\alpha t} \frac{1}{G} \sum_{g=1}^{G}\left(\sigma_{g}^{2}\right)^{-\alpha t} \\
& C_{3}(\alpha) \triangleq \ln \left(h_{G}^{\alpha}(t) h_{1}^{1-\alpha}(t)\right)
\end{aligned}
$$

where $C_{2}(\alpha)$ is the same as $b\left(\boldsymbol{\sigma}^{2}, \alpha t\right) . \quad A(\alpha)$ and $R_{1}\left(\boldsymbol{\sigma}^{2 t}, \hat{\boldsymbol{\sigma}}^{2 t}(\alpha)\right)$ differ only by a constant independent of $\alpha$. Let

$$
D(\alpha) \triangleq \ln \left(\frac{h_{G}(t)}{h_{1}(t)}\right)+\frac{(G-1) t}{G}\left[\Psi\left(\frac{\nu}{2}+\frac{\alpha t}{G}\right)-\Psi\left(\frac{\nu}{2}+\left(1-\alpha+\frac{\alpha}{G}\right) t\right)\right] .
$$

Then it is not difficult to check that

$$
\begin{aligned}
& C_{1}^{\prime}(\alpha)=C_{1}(\alpha) D(\alpha), \\
& C_{1}^{\prime \prime}(\alpha)=C_{1}(\alpha)\left(D^{2}(\alpha)+D^{\prime}(\alpha)\right), \\
& C_{2}^{\prime}(\alpha)=\left(\sigma_{\text {pool }}^{2}\right)^{\alpha t} \frac{1}{G} \sum_{g=1}^{G}\left[\left(\sigma_{g}^{2}\right)^{-\alpha t} t \ln \left(\frac{\sigma_{\text {pool }}^{2}}{\sigma_{g}^{2}}\right)\right], \\
& C_{2}^{\prime \prime}(\alpha)=\left(\sigma_{\text {pool }}^{2}\right)^{\alpha t} \frac{1}{G} \sum_{g=1}^{G}\left[\left(\sigma_{g}^{2}\right)^{-\alpha t} t^{2} \ln ^{2}\left(\frac{\sigma_{\text {pool }}^{2}}{\sigma_{g}^{2}}\right)\right],
\end{aligned}
$$


where

$$
D^{\prime}(\alpha)=\frac{(G-1) t^{2}}{G^{2}}\left[\Psi^{\prime}\left(\frac{\nu}{2}+\frac{\alpha t}{G}\right)+(G-1) \Psi^{\prime}\left(\frac{\nu}{2}+\left(1-\alpha+\frac{\alpha}{G}\right) t\right)\right] .
$$

For any $\alpha \in[0,1]$ and non-zero $t>-\nu / 2, \nu / 2+\alpha t / G>0$ and $\nu / 2+(1-\alpha+\alpha / G) t>0$. Thus by $(15), D^{\prime}(\alpha)>0$.

[Proof of $(i)]$

$$
\begin{aligned}
A^{\prime}(0) & =C_{1}^{\prime}(0) C_{2}(0)+C_{1}(0) C_{2}^{\prime}(0)-C_{3}^{\prime}(0) \\
& =\ln \left(\frac{h_{G}(t)}{h_{1}(t)}\right)+\frac{(G-1) t}{G}\left(\Psi\left(\frac{\nu}{2}\right)-\Psi\left(\frac{\nu}{2}+t\right)\right)-\ln \left(\frac{h_{G}(t)}{h_{1}(t)}\right) \\
& =\frac{(G-1) t}{G}\left(\Psi\left(\frac{\nu}{2}\right)-\Psi\left(\frac{\nu}{2}+t\right)\right) \\
& <0,
\end{aligned}
$$

where the last inequality holds for non-zero $t>-\nu / 2$ since $\Psi(\cdot)$ is a strictly increasing function on $(0, \infty)$.

[Proof of $(i i)]$

$$
\begin{aligned}
A^{\prime}(1) & =\ln \left(\frac{h_{G}(t)}{h_{1}(t)}\right)\left(\sigma_{\text {pool }}^{2}\right)^{t} \frac{1}{G} \sum_{g=1}^{G}\left(\sigma_{g}^{2}\right)^{-t}+\left(\sigma_{\text {pool }}^{2}\right)^{t} \frac{1}{G} \sum_{g=1}^{G}\left(\sigma_{g}^{2}\right)^{-t} t \ln \left(\frac{\sigma_{\text {pool }}^{2}}{\sigma_{g}^{2}}\right)-\ln \left(\frac{h_{G}(t)}{h_{1}(t)}\right) \\
& =\ln \left(\frac{h_{G}(t)}{h_{1}(t)}\right)\left(\left(\sigma_{\text {pool }}^{2}\right)^{t} \frac{1}{G} \sum_{g=1}^{G}\left(\sigma_{g}^{2}\right)^{-t}-1\right)+\left(\sigma_{\text {pool }}^{2}\right)^{t} \frac{1}{G} \sum_{g=1}^{G}\left(\sigma_{g}^{2}\right)^{-t} t \ln \left(\frac{\sigma_{\text {pool }}^{2}}{\sigma_{g}^{2}}\right) \\
& \triangleq \ln \left(h_{G}(t) / h_{1}(t)\right) M_{1}+M_{2},
\end{aligned}
$$

where $M_{1} \triangleq\left(\sigma_{\text {pool }}^{2}\right)^{t} \sum_{g=1}^{G}\left(\sigma_{g}^{2}\right)^{-t} / G-1 \geq\left(\sigma_{\text {pool }}^{2}\right)^{t}\left(\prod_{g=1}^{G} \sigma_{g}^{2}\right)^{-t / G}-1=0$, and

$$
\begin{aligned}
M_{2} & \triangleq\left(\sigma_{\text {pool }}^{2}\right)^{t} \frac{1}{G} \sum_{g=1}^{G}\left(\sigma_{g}^{2}\right)^{-t} t \ln \left(\sigma_{\text {pool }}^{2} / \sigma_{g}^{2}\right) \\
& =\left(\sigma_{\text {pool }}^{2}\right)^{t} \frac{1}{G} \sum_{g=1}^{G} \frac{1}{\left(\sigma_{g}^{2}\right)^{t}}\left(\frac{1}{G} \sum_{i=1}^{G} \ln \left(\sigma_{i}^{2}\right)^{t}-\ln \left(\sigma_{g}^{2}\right)^{t}\right) \\
& \geq 0,
\end{aligned}
$$

where the last inequality is a consequence of Lemma 4 . By Lemma 3 (iii), $\ln \left(h_{G}(t) / h_{1}(t)\right)>0$. Thus $A^{\prime}(1) \geq 0$. It is easy to see that equality holds iff $\sigma_{g}^{2}=\sigma^{2}$ for all $g$. 
$[$ Proof of $($ iii $)]$

$$
\begin{aligned}
A^{\prime \prime}(\alpha)= & C_{1}^{\prime \prime}(\alpha) C_{2}(\alpha)+2 C_{1}^{\prime}(\alpha) C_{2}^{\prime}(\alpha)+C_{1}(\alpha) C_{2}^{\prime \prime}(\alpha)-C_{3}^{\prime \prime}(\alpha) \\
= & C_{1}(\alpha)\left[D^{2}(\alpha)+D^{\prime}(\alpha)\right] C_{2}(\alpha)+2 C_{1}(\alpha) D(\alpha) C_{2}^{\prime}(\alpha)+C_{1}(\alpha) C_{2}^{\prime \prime}(\alpha) \\
= & C_{1}(\alpha)\left(\sigma_{\text {pool }}^{2}\right)^{\alpha t} \frac{1}{G}\left[\sum_{g=1}^{G}\left(\sigma_{g}^{2}\right)^{-\alpha t}\left(D^{2}(\alpha)+D^{\prime}(\alpha)\right)\right. \\
& \left.+\sum_{g=1}^{G}\left(\sigma_{g}^{2}\right)^{-\alpha t} 2 D(\alpha) t \ln \left(\frac{\sigma_{\text {pool }}^{2}}{\sigma_{g}^{2}}\right)+\sum_{g=1}^{G}\left(\sigma_{g}^{2}\right)^{-\alpha t} t^{2} \ln ^{2}\left(\frac{\sigma_{\text {pool }}^{2}}{\sigma_{g}^{2}}\right)\right] \\
= & C_{1}(\alpha)\left(\sigma_{\text {pool }}^{2}\right)^{\alpha t} \frac{1}{G} \sum_{g=1}^{G}\left(\sigma_{g}^{2}\right)^{-\alpha t}\left[\left(D(\alpha)+t \ln \left(\sigma_{\text {pool }}^{2} / \sigma_{g}^{2}\right)\right)^{2}+D^{\prime}(\alpha)\right] \\
> & 0,
\end{aligned}
$$

where the last inequality uses the fact that $D^{\prime}(\alpha)>0$ for any $\alpha \in[0,1]$.

\section{APPENDIX 2: THE PROOF OF THEOREM 2}

[Proof of $(i)]$ For any fixed $t>0$, let $m$ be the smallest integer such that $m \geq t$. By (14) and the fact that $\Psi(\cdot)$ is an increasing function on $(0, \infty)$, we have

$$
0 \geq \Psi\left(\frac{\nu}{2}\right)-\Psi\left(\frac{\nu}{2}+t\right) \geq \Psi\left(\frac{\nu}{2}\right)-\Psi\left(\frac{\nu}{2}+m\right)=-\sum_{k=0}^{m-1} \frac{1}{\nu / 2+k} \geq-\frac{2 m}{\nu} \stackrel{\nu \rightarrow \infty}{\longrightarrow} 0 .
$$

Thus by $(22)$, for any fixed $G$ and $t, \lim _{\nu \rightarrow \infty} A^{\prime}(0)=0$. Similar arguments show that $\lim _{\nu \rightarrow \infty} A^{\prime}(0)=0$ for any fixed $-\nu / 2<t<0$.

Lemma $3(v)$ implies that $\lim _{\nu \rightarrow \infty} C_{1}(\alpha)=1$ for any fixed $\alpha, G$ and $t$. Similar arguments as in (24) show that $\lim _{\nu \rightarrow \infty} D(\alpha)=0$. By (15) it is easy to see that $\lim _{\nu \rightarrow \infty} D^{\prime}(\alpha)=\lim _{\nu \rightarrow \infty} 2(1-1 / G) t^{2} / \nu=0$. Thus,

$$
\lim _{\nu \rightarrow \infty} A^{\prime \prime}(\alpha)=\left(\sigma_{\text {pool }}^{2}\right)^{\alpha t} \frac{1}{G} \sum_{g=1}^{G}\left(\sigma_{g}^{2}\right)^{-\alpha t} t^{2} \ln ^{2}\left(\frac{\sigma_{\text {pool }}^{2}}{\sigma_{g}^{2}}\right) .
$$

When $\sigma_{g}^{2}$ are not all the same, $\lim _{\nu \rightarrow \infty} A^{\prime \prime}(\alpha)>0$. This shows that $A(\alpha)$ reaches the minimum at $\alpha=0$ when $\nu \rightarrow \infty$ since $\lim _{\nu \rightarrow \infty} A^{\prime}(0)=0$ and $A(\alpha)$ is a strictly convex function of $\alpha$ in $[0,1]$.

[Proof of $(i i)]$ By $(25), \lim _{\nu \rightarrow \infty} A^{\prime \prime}(\alpha)=0$ when $\sigma_{g}^{2}=\sigma^{2}$ for all $g$. Combining with the fact that $\lim _{\nu \rightarrow \infty} A^{\prime}(0)=0, A(\alpha)$ is a constant function of $\alpha$ in $[0,1]$.

\section{APPENDIX 3: THE PROOF OF THEOREM 3}

Lemma 5. For any $s>0$ and $t>-s$, we have

$$
\ln (\Gamma(s+t) / \Gamma(s)) \geq t \Psi(s) .
$$


[Proof] Let $f(t)=\ln (\Gamma(s+t) / \Gamma(s))-t \Psi(s)$ and we need to show that $f(t) \geq 0$. $f^{\prime}(t)=$ $\Psi(s+t)-\Psi(s)$. Since $\Psi(\cdot)$ is an increasing function, $f^{\prime}(t) \geq 0$ on $(0, \infty)$ and $f^{\prime}(t) \leq 0$ on $(-s, 0)$. Thus $f(t) \geq f(0)=0$.

Proof of Theorem 3. We rewrite (9) as

$$
R_{2}\left(\boldsymbol{\sigma}^{2 t}, \hat{\boldsymbol{\sigma}}^{2 t}\right) \triangleq E_{1}(\alpha) E_{2}(\alpha)-2 C_{1}(\alpha) C_{2}(\alpha)+G,
$$

where $C_{1}(\alpha)$ and $C_{2}(\alpha)$ are defined in (18) and (19) and

$$
\begin{aligned}
& E_{1}(\alpha) \triangleq \frac{h_{G}^{2 \alpha}(t) h_{1}^{2(1-\alpha)}(t)}{h_{1}^{G-1}\left(\frac{2 \alpha t}{G}\right) h_{1}\left(2\left(1-\alpha+\frac{\alpha}{G}\right) t\right)}, \\
& E_{2}(\alpha) \triangleq\left(\sigma_{\text {pool }}^{2}\right)^{2 \alpha t} \frac{1}{G} \sum_{g=1}^{G}\left(\sigma_{g}^{2}\right)^{-2 \alpha t} .
\end{aligned}
$$

$[$ Proof of $(i)]$

$$
\begin{aligned}
\left.R_{2}^{\prime}\left(\boldsymbol{\sigma}^{2 t}, \hat{\boldsymbol{\sigma}}^{2 t}\right)\right|_{\alpha=0}= & 2\left(\frac{h_{1}^{2}(t)}{h_{1}(2 t)}-1\right)\left[\ln \left(\frac{h_{G}(t)}{h_{1}(t)}\right)+\frac{(G-1) t}{G}\left(\Psi\left(\frac{\nu}{2}\right)-\Psi\left(\frac{\nu}{2}+t\right)\right)\right] \\
& +2 \frac{h_{1}^{2}(t)}{h_{1}(2 t)} \frac{(G-1) t}{G}\left[\Psi\left(\frac{\nu}{2}\right)-\Psi\left(\frac{\nu}{2}+t\right)\right] \\
< & 2\left(\frac{h_{1}^{2}(t)}{h_{1}(2 t)}-1\right)\left[\ln \left(\frac{h_{G}(t)}{h_{1}(t)}\right)+\frac{(G-1) t}{G}\left(\Psi\left(\frac{\nu}{2}\right)-\Psi\left(\frac{\nu}{2}+t\right)\right)\right] \\
\triangleq & 2 M_{3} M_{4},
\end{aligned}
$$

where the inequality holds following the same arguments in the proof of Theorem 1 (i). By Lemma 3(ii), $\Gamma(\nu / 2) / \Gamma(\nu / 2+t) \geq \Gamma(\nu / 2+t) / \Gamma(\nu / 2+2 t)$ for any $t>-\nu / 2$ and $t \neq 0$. Thus $h_{1}^{2}(t) \geq h_{1}(2 t)$ which implies that $M_{3} \geq 0$. By Lemma 5 ,

$$
\begin{aligned}
-\ln \left(\frac{h_{G}(t)}{h_{1}(t)}\right) & =(G-1) \ln \left(\frac{\Gamma\left(\frac{\nu}{2}+\frac{t}{G}\right)}{\Gamma\left(\frac{\nu}{2}\right)}\right)+\ln \left(\frac{\Gamma\left(\frac{\nu}{2}+\frac{t}{G}\right)}{\Gamma\left(\frac{\nu}{2}+t\right)}\right) \\
& \geq(G-1) \frac{t}{G} \Psi\left(\frac{\nu}{2}\right)-\frac{(G-1) t}{G} \Psi\left(\frac{\nu}{2}+t\right) \\
& =\frac{(G-1) t}{G}\left(\Psi\left(\frac{\nu}{2}\right)-\Psi\left(\frac{\nu}{2}+t\right)\right),
\end{aligned}
$$

which implies that $M_{4} \leq 0$. Therefore, $\left.R_{2}^{\prime}\left(\boldsymbol{\sigma}^{2 t}, \hat{\boldsymbol{\sigma}}^{2 t}\right)\right|_{\alpha=0}<0$.

[Proof of $(i i)]$ It is easy to check that $\left.R_{2}^{\prime}\left(\boldsymbol{\sigma}^{2 t}, \hat{\boldsymbol{\sigma}}^{2 t}\right)\right|_{\alpha=1} \triangleq\left(M_{5}+M_{6}\right) / G$, where

$$
\begin{aligned}
& M_{5} \triangleq 2 \ln \left(\frac{h_{G}(t)}{h_{1}(t)}\right)\left(\frac{h_{G}^{2}(t)}{h_{1}^{G}\left(\frac{2 t}{G}\right)}\left(\sigma_{\text {pool }}^{2}\right)^{2 t} \sum_{g=1}^{G}\left(\sigma_{g}^{2}\right)^{-2 t}-\left(\sigma_{\text {pool }}^{2}\right)^{t} \sum_{g=1}^{G}\left(\sigma_{g}^{2}\right)^{-t}\right), \\
& M_{6} \triangleq \frac{h_{G}^{2}(t)}{h_{1}^{G}\left(\frac{2 t}{G}\right)}\left(\sigma_{\text {pool }}^{2}\right)^{2 t} \sum_{g=1}^{G}\left[\left(\sigma_{g}^{2}\right)^{-2 t} 2 t \ln \left(\frac{\sigma_{\text {pool }}^{2}}{\sigma_{g}^{2}}\right)\right]-\left(\sigma_{\text {pool }}^{2}\right)^{t} \sum_{g=1}^{G}\left[\left(\sigma_{g}^{2}\right)^{-t} 2 t \ln \left(\frac{\sigma_{\text {pool }}^{2}}{\sigma_{g}^{2}}\right)\right] .
\end{aligned}
$$


We will show that $M_{5}>0$ and $M_{6} \geq 0$. Using the facts that $\left(\sigma_{\text {pool }}^{2}\right)^{t} \sum_{g=1}^{G}\left(\sigma_{g}^{2}\right)^{-t} \geq G$ and $\left(\sigma_{\text {pool }}^{2}\right)^{2 t} \sum_{g=1}^{G}\left(\sigma_{g}^{2}\right)^{-2 t}-2\left(\sigma_{\text {pool }}^{2}\right)^{t} \sum_{g=1}^{G}\left(\sigma_{g}^{2}\right)^{-t}+G=\sum_{g=1}^{G}\left(\left(\sigma_{\text {pool }}^{2} / \sigma_{g}^{2}\right)^{t}-1\right)^{2} \geq 0$, we have $\left(\sigma_{\text {pool }}^{2}\right)^{2 t} \sum_{g=1}^{G}\left(\sigma_{g}^{2}\right)^{-2 t} \geq\left(\sigma_{\text {pool }}^{2}\right)^{t} \sum_{g=1}^{G}\left(\sigma_{g}^{2}\right)^{-t}$. Using Lemma 3 (iii) and (iv),

$$
M_{5}>2 \ln \left(\frac{h_{G}(t)}{h_{1}(t)}\right)\left(\left(\sigma_{\text {pool }}^{2}\right)^{2 t} \sum_{g=1}^{G}\left(\sigma_{g}^{2}\right)^{-2 t}-\left(\sigma_{\text {pool }}^{2}\right)^{t} \sum_{g=1}^{G}\left(\sigma_{g}^{2}\right)^{-t}\right) \geq 0 .
$$

By Lemma 3 (iv) and Lemma 4,

$$
\begin{aligned}
M_{6} & \geq\left(\sigma_{\text {pool }}^{2}\right)^{2 t} \sum_{g=1}^{G}\left[\left(\sigma_{g}^{2}\right)^{-2 t} 2 t \ln \left(\frac{\sigma_{\text {pool }}^{2}}{\sigma_{g}^{2}}\right)\right]-\left(\sigma_{\text {pool }}^{2}\right)^{t} \sum_{g=1}^{G}\left[\left(\sigma_{g}^{2}\right)^{-t} 2 t \ln \left(\frac{\sigma_{\text {pool }}^{2}}{\sigma_{g}^{2}}\right)\right] \\
& =\sum_{g=1}^{G} 2 \ln \left(\frac{\sigma_{\text {pool }}^{2}}{\sigma_{g}^{2}}\right)^{t}\left[\left(\frac{\sigma_{\text {pool }}^{2}}{\sigma_{g}^{2}}\right)^{2 t}-\left(\frac{\sigma_{\text {pool }}^{2}}{\sigma_{g}^{2}}\right)^{t}\right] \\
& \geq 0
\end{aligned}
$$

where the last inequality uses the fact that $\ln (x)$ and $x(x-1)$ have the same sign for all $x>0$.

\section{APPENDIX 4: COUNTER EXAMPLE AND PROOF OF THEOREM 4}

Some algebra shows that

$$
\begin{aligned}
R_{2}^{\prime \prime}\left(\boldsymbol{\sigma}^{2 t}, \hat{\boldsymbol{\sigma}}^{2 t}\right) & =E_{1}(\alpha)\left(\sigma_{\text {pool }}^{2}\right)^{2 \alpha t} \frac{1}{G} \sum_{g=1}^{G}\left(\sigma_{g}^{2}\right)^{-2 \alpha t}\left\{\left[F(\alpha)+2 t \ln \left(\frac{\sigma_{\text {pool }}^{2}}{\sigma_{g}^{2}}\right)\right]^{2}+F^{\prime}(\alpha)\right\} \\
& -2 C_{1}(\alpha)\left(\sigma_{\text {pool }}^{2}\right)^{\alpha t} \frac{1}{G} \sum_{g=1}^{G}\left(\sigma_{g}^{2}\right)^{-\alpha t}\left\{\left[D(\alpha)+t \ln \left(\frac{\sigma_{\text {pool }}^{2}}{\sigma_{g}^{2}}\right)\right]^{2}+D^{\prime}(\alpha)\right\}
\end{aligned}
$$

where $C_{1}(\alpha), D(\alpha), D^{\prime}(\alpha)$ and $E_{1}(\alpha)$ are defined in (18), (20), (21) and (26) respectively, and

$$
\begin{aligned}
& F(\alpha) \triangleq 2 \ln \left(\frac{h_{G}(t)}{h_{1}(t)}\right)+\frac{2(G-1) t}{G}\left[\Psi\left(\frac{\nu}{2}+\frac{2 \alpha t}{G}\right)-\Psi\left(\frac{\nu}{2}+2\left(1-\alpha+\frac{\alpha}{G}\right) t\right)\right], \\
& F^{\prime}(\alpha) \triangleq \frac{4(G-1) t^{2}}{G^{2}}\left[\Psi^{\prime}\left(\frac{\nu}{2}+\frac{2 \alpha t}{G}\right)+(G-1) \Psi^{\prime}\left(\frac{\nu}{2}+2\left(1-\alpha+\frac{\alpha}{G}\right) t\right)\right] .
\end{aligned}
$$

We have shown in Appendix 2 that $\lim _{\nu \rightarrow \infty} C_{1}(\alpha)=1$ and $\lim _{\nu \rightarrow \infty} D(\alpha)=\lim _{\nu \rightarrow \infty} D^{\prime}(\alpha)=0$. Similar arguments show that for any fixed $\alpha, G$ and $t, \lim _{\nu \rightarrow \infty} E_{1}(\alpha)=1$ and $\lim _{\nu \rightarrow \infty} F(\alpha)=\lim _{\nu \rightarrow \infty} F^{\prime}(\alpha)=0$. Denote $a_{g} \triangleq\left(\sigma_{\text {pool }}^{2} / \sigma_{g}^{2}\right)^{t}$. We have $\prod_{g=1}^{G} a_{g}=1$, and

$$
\lim _{\nu \rightarrow \infty} R_{2}^{\prime \prime}\left(\boldsymbol{\sigma}^{2 t}, \hat{\boldsymbol{\sigma}}^{2 t}\right)=\frac{2}{G} \sum_{g=1}^{G}\left(2 a_{g}^{2 \alpha}-a_{g}^{\alpha}\right)\left(\ln a_{g}\right)^{2} .
$$


[Counter Example] Let $G=100, a_{1}=\cdots=a_{99}=16^{1 / 99}$ and $a_{100}=1 / 16$. It is easy to check that $\prod_{g=1}^{G} a_{g}=1$ and $\left.\lim _{\nu \rightarrow \infty} R_{2}^{\prime \prime}\left(\boldsymbol{\sigma}^{2 t}, \hat{\boldsymbol{\sigma}}^{2 t}\right)\right|_{\alpha=0.5}=-0.0176<0$. Therefore, there exists a large $\nu$ such that $R_{2}\left(\boldsymbol{\sigma}^{2 t}, \hat{\boldsymbol{\sigma}}^{2 t}\right)$ is not a convex function.

[Proof of Theorem 4] Similar arguments as in (29) show that for any $\alpha \in[0,1]$,

$$
\lim _{\nu \rightarrow \infty} R_{2}^{\prime}\left(\boldsymbol{\sigma}^{2 t}, \hat{\boldsymbol{\sigma}}^{2 t}\right)=\frac{2}{G} \sum_{g=1}^{G}\left(a_{g}^{2 \alpha}-a_{g}^{\alpha}\right) \ln \left(a_{g}\right)=\frac{2}{\alpha G} \sum_{g=1}^{G} a_{g}^{\alpha}\left(a_{g}^{\alpha}-1\right) \ln \left(a_{g}^{\alpha}\right) \geq 0,
$$

where the equality holds iff $a_{g}=1$ for all $g$ in the last inequality. This implies that $\alpha_{2}^{*} \rightarrow 0$ as $\nu \rightarrow \infty$ when $\sigma_{g}^{2}$ are not all the same. Otherwise, $\lim _{\nu \rightarrow \infty} R_{2}^{\prime}\left(\boldsymbol{\sigma}^{2 t}, \hat{\boldsymbol{\sigma}}^{2 t}\right)=0$ which proves part $(i i)$.

\section{APPENDIX 5: THE PROOF OF THEOREM 5}

It is easy to see that $\left(Z_{g} / \sigma_{g}^{2}\right)^{-t} \stackrel{a . s .}{\longrightarrow} 1$ for any fixed $t$. For an arbitrary $\epsilon>0$, there exists an $N>0$ such that for any $\nu>N, 1-\epsilon \leq\left(Z_{g} / \sigma_{g}^{2}\right)^{-t} \leq 1+\epsilon$ with probability 1 . Now for any $\alpha \in[0,1], 1-\epsilon \leq(1-\epsilon)^{\alpha} \leq\left(Z_{g} / \sigma_{g}^{2}\right)^{-\alpha t} \leq(1+\epsilon)^{\alpha} \leq 1+\epsilon$. Therefore, $Z_{g}^{-\alpha t} \stackrel{a . s .}{\longrightarrow}$ $\left(\sigma_{g}^{2}\right)^{-\alpha t}$ uniformly for $\alpha \in[0,1]$ as $\nu \rightarrow \infty$. Note that $G$ is fixed, we have

$$
\frac{1}{G} \sum_{g=1}^{G} Z_{g}^{-\alpha t} \stackrel{a . s .}{\longrightarrow} \frac{1}{G} \sum_{g=1}^{G}\left(\sigma_{g}^{2}\right)^{-\alpha t} \text { uniformly for } \alpha \in[0,1] \text { as } \nu \rightarrow \infty .
$$

Similarly, by the fact that $Z_{\text {pool }} / \sigma_{\text {pool }}^{2} \stackrel{a . s .}{\longrightarrow} 1$, we have

$$
\left(Z_{\text {pool }}\right)^{\alpha t} \stackrel{\text { a.s. }}{\longrightarrow}\left(\sigma_{\text {pool }}^{2}\right)^{\alpha t} \text { uniformly for } \alpha \in[0,1] \text { as } \nu \rightarrow \infty \text {. }
$$

Thus by (30) and (31) and the facts that $\left(\sigma_{\text {pool }}^{2}\right)^{\alpha t}<\infty$ and $\sum_{g=1}^{G}\left(\sigma_{g}^{2}\right)^{-\alpha t} / G<\infty$,

$$
\begin{aligned}
b(\mathbf{Z}, \alpha t)= & \left(\left(Z_{\text {pool }}\right)^{\alpha t}-\left(\sigma_{\text {pool }}^{2}\right)^{\alpha t}\right)\left(\frac{1}{G} \sum_{g=1}^{G} Z_{g}^{-\alpha t}-\frac{1}{G} \sum_{g=1}^{G}\left(\sigma_{g}^{2}\right)^{-\alpha t}\right) \\
& +\left(\sigma_{\text {pool }}^{2}\right)^{\alpha t}\left(\frac{1}{G} \sum_{g=1}^{G} Z_{g}^{-\alpha t}-\frac{1}{G} \sum_{g=1}^{G}\left(\sigma_{g}^{2}\right)^{-\alpha t}\right) \\
& +\left(\left(Z_{\text {pool }}\right)^{\alpha t}-\left(\sigma_{\text {pool }}^{2}\right)^{\alpha t}\right) \frac{1}{G} \sum_{g=1}^{G}\left(\sigma_{g}^{2}\right)^{-\alpha t} \\
& +\left(\sigma_{\text {pool }}^{2}\right)^{\alpha t} \frac{1}{G} \sum_{g=1}^{G}\left(\sigma_{g}^{2}\right)^{-\alpha t} \\
\stackrel{\text { a.s. }}{\longrightarrow} & b\left(\boldsymbol{\sigma}^{2}, \alpha t\right) \text { uniformly for } \alpha \in[0,1] \text { as } \nu \rightarrow \infty .
\end{aligned}
$$

For a fixed $t$, let $Q_{k}\left(\boldsymbol{\sigma}^{2}, \alpha, \nu\right)=R_{k}\left(\boldsymbol{\sigma}^{2 t}, \hat{\boldsymbol{\sigma}}^{2 t}(\alpha)\right), Q_{k}(\mathbf{Z}, \alpha, \nu)$ be the functions with $b\left(\boldsymbol{\sigma}^{2}, k \alpha t\right)$ in $Q_{k}\left(\boldsymbol{\sigma}^{2}, \alpha, \nu\right)$ replaced by $b(\mathbf{Z}, k \alpha t), k=1,2$. By $(32)$, it is not difficult to check that

$$
Q_{1}(\mathbf{Z}, \alpha, \nu)-Q_{1}\left(\boldsymbol{\sigma}^{2}, \alpha, \nu\right) \stackrel{\text { a.s. }}{\longrightarrow} 0 \text { uniformly for } \alpha \in[0,1] \text { as } \nu \rightarrow \infty \text {. }
$$


Then for each pair $\left(\boldsymbol{\sigma}^{2}, \mathbf{Z}\right)$ satisfying (33), it is easy to show that $\min _{\alpha \in[0,1]} Q_{1}(\mathbf{Z}, \alpha, \nu)-\min _{\alpha \in[0,1]} Q_{1}\left(\boldsymbol{\sigma}^{2}, \alpha, \nu\right) \rightarrow$ 0. Note that $\min _{\alpha \in[0,1]} Q_{1}\left(\boldsymbol{\sigma}^{2}, \alpha, \nu\right)=R_{1}\left(\boldsymbol{\sigma}^{2 t}, \hat{\boldsymbol{\sigma}}^{2 t}\left(\alpha_{1}^{*}(\nu)\right)\right)$ and

$$
\begin{aligned}
& \min _{\alpha \in[0,1]} Q_{1}(\mathbf{Z}, \alpha, \nu)-R_{1}\left(\boldsymbol{\sigma}^{2 t}, \hat{\boldsymbol{\sigma}}^{2 t}\left(\hat{\alpha}_{1}^{*}(\nu)\right)\right) \\
= & C_{1}\left(\hat{\alpha}_{1}^{*}(\nu)\right)\left[b\left(\mathbf{Z}, \hat{\alpha}_{1}^{*}(\nu) t\right)-b\left(\boldsymbol{\sigma}^{2}, \hat{\alpha}_{1}^{*}(\nu) t\right)\right] \\
\rightarrow & 0 \text { as } \nu \rightarrow \infty,
\end{aligned}
$$

since $\lim _{\nu \rightarrow \infty} C_{1}(\alpha)=1$. Therefore,

$$
R_{1}\left(\boldsymbol{\sigma}^{2 t}, \hat{\boldsymbol{\sigma}}^{2 t}\left(\hat{\alpha}_{1}^{*}\right)\right)-R_{1}\left(\boldsymbol{\sigma}^{2 t}, \hat{\boldsymbol{\sigma}}^{2 t}\left(\alpha_{1}^{*}\right)\right) \stackrel{\text { a.s. }}{\longrightarrow} 0 \text { as } \nu \rightarrow \infty .
$$

Similar arguments show that (34) holds for the squared loss function. From (34) we have $b\left(\boldsymbol{\sigma}^{2}, \hat{\alpha}_{1}^{*} t\right)-b\left(\boldsymbol{\sigma}^{2}, \alpha_{1}^{*} t\right) \stackrel{\text { a.s. }}{\rightarrow} 0$. Note that $\alpha_{1}^{*} \rightarrow 0$ since $\sigma_{g}^{2}$ are not all the same (Theorem 2$)$. This implies that $b\left(\boldsymbol{\sigma}^{2}, \alpha_{1}^{*} t\right) \rightarrow b\left(\boldsymbol{\sigma}^{2}, 0\right)=1$ and thus $b\left(\boldsymbol{\sigma}^{2}, \hat{\alpha}_{1}^{*} t\right) \stackrel{\text { a.s. }}{\rightarrow} 1$. Therefore, by the fact that $\sum_{g=1}^{G}\left(\sigma_{g}^{2}\right)^{p} / G>\left(\sigma_{\text {pool }}^{2}\right)^{p}$ for any non-zero $p$ since $\sigma_{g}^{2}$ are not all the same, we have $\hat{\alpha}_{1}^{*} \stackrel{a . s .}{\rightarrow} 0$.

Similarly, we have $\left[b\left(\boldsymbol{\sigma}^{2}, 2 \hat{\alpha}_{2}^{*} t\right)-b\left(\boldsymbol{\sigma}^{2}, 2 \alpha_{2}^{*} t\right)\right]-2\left[b\left(\boldsymbol{\sigma}^{2}, \hat{\alpha}_{2}^{*} t\right)-b\left(\boldsymbol{\sigma}^{2}, \alpha_{2}^{*} t\right)\right] \stackrel{\text { a.s. }}{\rightarrow} 0$. Thus $\hat{\alpha}_{2}^{*} \stackrel{a . s .}{\rightarrow} 0$ by noting that $\alpha_{2}^{*} \rightarrow 0$ (Theorem 4 ) and the fact that

$$
b\left(\boldsymbol{\sigma}^{2}, 2 \hat{\alpha}_{2}^{*} t\right)-2 b\left(\boldsymbol{\sigma}^{2}, \hat{\alpha}_{2}^{*} t\right)+1=\frac{1}{G} \sum_{g=1}^{G}\left[\left(\sigma_{\text {pool }}^{2} / \sigma_{g}^{2}\right)^{\hat{\alpha}_{2}^{*} t}-1\right]^{2} \geq 0 .
$$

\section{APPENDIX 6: THE PROOF OF LEMMA 2 AND THEOREM 6}

[Proof of Lemma 2] Since $s(\alpha)=\left(\sigma_{g}^{2}\right)^{-\alpha t}$ is a convex function of $\alpha$, then $\left(\sigma_{g}^{2}\right)^{-\alpha t}=s(\alpha) \leq$ $(1-\alpha) s(0)+\alpha s(1) \leq 1+\left(\sigma_{g}^{2}\right)^{-t}$. By Theorem 16(a) in Ferguson (1996) and the fact that $\mathrm{E}\left(\sigma_{g}^{2}\right)^{-t}<\infty$

$$
\frac{1}{G} \sum_{g=1}^{G}\left(\sigma_{g}^{2}\right)^{-\alpha t} \stackrel{\text { a.s. }}{\longrightarrow} \mathrm{E}\left(\sigma_{1}^{2}\right)^{-\alpha t} \text { uniformly for } \alpha \in[0,1] \text { as } G \rightarrow \infty .
$$

Similarly, by the fact that $\mathrm{E}\left(Z_{1}\right)^{-\alpha t}=\mathrm{E}\left[\mathrm{E}\left(Z_{1}^{-\alpha t} \mid \sigma_{1}^{2}\right)\right]=\mathrm{E}\left(\sigma_{1}^{2}\right)^{-\alpha t} / h_{1}(-\alpha t)$,

$$
\frac{h_{1}(-\alpha t)}{G} \sum_{g=1}^{G}\left(Z_{g}\right)^{-\alpha t} \stackrel{\text { a.s. }}{\longrightarrow} \mathrm{E}\left(\sigma_{1}^{2}\right)^{-\alpha t} \text { uniformly for } \alpha \in[0,1] \text { as } G \rightarrow \infty .
$$

Combining (35) and (36),

$$
\frac{h_{1}(-\alpha t)}{G} \sum_{g=1}^{G}\left(Z_{g}\right)^{-\alpha t}-\frac{1}{G} \sum_{g=1}^{G}\left(\sigma_{g}^{2}\right)^{-\alpha t} \stackrel{a . s .}{\longrightarrow} 0 \text { uniformly for } \alpha \in[0,1] \text { as } G \rightarrow \infty .
$$


By Strong law of large numbers, $\ln \left(\sigma_{\text {pool }}^{2}\right)=\sum_{g=1}^{G} \ln \left(\sigma_{g}^{2}\right) / G \stackrel{\text { a.s. }}{\rightarrow} \mathrm{E} \ln \left(\sigma_{1}^{2}\right)$. Thus $\left[\sigma_{\text {pool }}^{2} \exp \left(-\mathrm{E} \ln \left(\sigma_{1}^{2}\right)\right)\right]^{t} \stackrel{\text { a.s. }}{\rightarrow}$ 1 for any fixed $t$. Thus by the similar arguments as in (30),

$$
\left(\sigma_{\text {pool }}^{2}\right)^{\alpha t} \stackrel{\text { a.s. }}{\longrightarrow} \exp \left(\alpha t \mathrm{E} \ln \left(\sigma_{1}^{2}\right)\right) \text { uniformly for } \alpha \in[0,1] \text { as } G \rightarrow \infty .
$$

Following the similar arguments and using the fact that $\mathrm{E}\left(\ln \left(Z_{1}\right)\right)=\mathrm{E}\left[\mathrm{E}\left(\ln \left(Z_{1}\right) \mid \sigma_{1}^{2}\right)\right]=$ $\mathrm{E} \ln \left(\sigma_{1}^{2}\right)+\Psi(\nu / 2)-\ln (\nu / 2)$, we have

$$
\left(\frac{\nu}{2}\right)^{\alpha t} \exp \left(-\alpha t \Psi\left(\frac{\nu}{2}\right)\right)\left(Z_{\text {pool }}\right)^{\alpha t} \stackrel{\text { a.s. }}{\longrightarrow} \exp \left[\alpha t \mathrm{E} \ln \left(\sigma_{1}^{2}\right)\right] \text { uniformly for } \alpha \in[0,1] \text { as } G \rightarrow \infty .
$$

Combining (38) and (39),

$$
\left(\frac{\nu}{2}\right)^{\alpha t} \exp \left(-\alpha t \Psi\left(\frac{\nu}{2}\right)\right)\left(Z_{\text {pool }}\right)^{\alpha t}-\left(\sigma_{\text {pool }}^{2}\right)^{\alpha t} \stackrel{\text { a.s. }}{\longrightarrow} 0 \text { uniformly for } \alpha \in[0,1] \text { as } G \rightarrow \infty .
$$

Using (36), (37), (38) and (40), we have

$$
\begin{aligned}
& w(\alpha t) b(\mathbf{Z}, \alpha t)-b\left(\boldsymbol{\sigma}^{2}, \alpha t\right) \\
= & {\left[\left(\frac{\nu}{2}\right)^{\alpha t} \exp \left(-\alpha t \Psi\left(\frac{\nu}{2}\right)\right)\left(Z_{\text {pool }}\right)^{\alpha t}-\left(\sigma_{\text {pool }}^{2}\right)^{\alpha t}\right] \frac{h_{1}(-\alpha t)}{G} \sum_{g=1}^{G}\left(Z_{g}\right)^{-\alpha t} } \\
& -\left(\sigma_{\text {pool }}^{2}\right)^{\alpha t}\left[\frac{h_{1}(-\alpha t)}{G} \sum_{g=1}^{G}\left(Z_{g}\right)^{-\alpha t}-\frac{1}{G} \sum_{g=1}^{G}\left(\sigma_{g}^{2}\right)^{-\alpha t}\right]
\end{aligned}
$$

$\stackrel{\text { a.s. }}{\longrightarrow} 0$ uniformly for $\alpha \in[0,1]$ as $G \rightarrow \infty$.

[Proof of Theorem $6(i)]$ By Lemma 2, it is not difficult to check that

$$
H_{1}(\mathbf{Z}, \alpha, G)-H_{1}\left(\boldsymbol{\sigma}^{2}, \alpha, G\right) \stackrel{\text { a.s. }}{\longrightarrow} 0 \text { uniformly for } \alpha \in[0,1] \text { as } G \rightarrow \infty .
$$

Similar arguments as in the proof of (34) together with the fact that $\max _{\alpha \in[0,1] G \rightarrow \infty} \lim _{G \rightarrow \infty} C_{1}(\alpha)<\infty$ lead to $R_{1}\left(\boldsymbol{\sigma}^{2 t}, \hat{\boldsymbol{\sigma}}^{2 t}\left(\breve{\alpha}_{1}^{*}(G)\right)\right)-R_{1}\left(\boldsymbol{\sigma}^{2 t}, \hat{\boldsymbol{\sigma}}^{2 t}\left(\alpha_{1}^{*}(G)\right)\right) \stackrel{\text { a.s. }}{\rightarrow} 0$.

Now we show for each pair $\left(\boldsymbol{\sigma}^{2}, \mathbf{Z}\right)$ satisfying $(41), \breve{\alpha}_{1}^{*}(G)-\alpha_{1}^{*}(G) \rightarrow 0$. Suppose that there exists a pair $\left(\boldsymbol{\sigma}^{2}, \mathbf{Z}\right)$ for which $(41)$ holds and $\breve{\alpha}_{1}^{*}(G)-\alpha_{1}^{*}(G) \nrightarrow 0$. Since $\alpha$ belongs to the compact interval $[0,1]$, there exists a subsequence $\left\{G_{n}\right\}$ such that $\left|\breve{\alpha}_{1}^{*}\left(G_{n}\right)-\alpha_{1}^{*}\left(G_{n}\right)\right| \rightarrow \beta>0$. Note that $\left(\sigma_{\text {pool }}^{2}\right)^{\alpha t} \sum_{g=1}^{G}\left(\sigma_{g}^{2}\right)^{-\alpha t} / G \geq 1$ for any $G$, by (23) we have

$$
\varliminf_{G \rightarrow \infty} \frac{\partial^{2} H_{1}\left(\boldsymbol{\sigma}^{2}, \alpha, G\right)}{\partial \alpha^{2}} \geq \lim _{G \rightarrow \infty} C_{1}(\alpha) D^{\prime}(\alpha)=\frac{\Gamma\left(\frac{\nu}{2}+(1-\alpha) t\right)}{\Gamma^{\alpha}\left(\frac{\nu}{2}\right) \Gamma^{1-\alpha}\left(\frac{\nu}{2}+t\right)} t^{2} \Psi^{\prime}\left(\frac{\nu}{2}+(1-\alpha) t\right) .
$$

Since the non-zero $t>-\nu / 2$, it is not difficult to show that $\delta \triangleq \min _{\alpha \in[0,1]}\left\{\lim _{G \rightarrow \infty} C_{1}(\alpha) D^{\prime}(\alpha)\right\}>0$. Then for an arbitrary $0<\epsilon<\delta \beta^{2} / 16$, there exists an $N_{1}>0$ such that for any $G_{n}>N_{1}$, 
$\left|\breve{\alpha}_{1}^{*}\left(G_{n}\right)-\alpha_{1}^{*}\left(G_{n}\right)\right|>\beta / 2$. Consequently,

$$
\begin{aligned}
& H_{1}\left(\boldsymbol{\sigma}^{2}, \breve{\alpha}_{1}^{*}\left(G_{n}\right), G_{n}\right)-H_{1}\left(\boldsymbol{\sigma}^{2}, \alpha_{1}^{*}\left(G_{n}\right), G_{n}\right) \\
= & \left.\frac{1}{2} \frac{\partial^{2} H_{1}\left(\boldsymbol{\sigma}^{2}, \alpha, G_{n}\right)}{\partial \alpha^{2}}\right|_{\alpha=\xi}\left(\breve{\alpha}_{1}^{*}\left(G_{n}\right)-\alpha_{1}^{*}\left(G_{n}\right)\right)^{2} \\
\geq & \frac{\delta \beta^{2}}{8} .
\end{aligned}
$$

For the same $\epsilon$, by (41), there exists another $N_{2}>0$ such that for any $G_{n}>N_{2}, \sup _{\alpha \in[0,1]} \mid H_{1}\left(\mathbf{Z}, \alpha, G_{n}\right)-$ $H_{1}\left(\boldsymbol{\sigma}^{2}, \alpha, G_{n}\right) \mid<\epsilon$. Therefore for any $G_{n}>\max \left\{N_{1}, N_{2}\right\}$, we have

$$
\begin{aligned}
H_{1}\left(\mathbf{Z}, \breve{\alpha}_{1}^{*}\left(G_{n}\right), G_{n}\right) & \geq H_{1}\left(\boldsymbol{\sigma}^{2}, \breve{\alpha}_{1}^{*}\left(G_{n}\right), G_{n}\right)-\epsilon \\
& \geq H_{1}\left(\boldsymbol{\sigma}^{2}, \alpha_{1}^{*}\left(G_{n}\right), G_{n}\right)+\delta \beta^{2} / 8-\epsilon \\
& \geq H_{1}\left(\mathbf{Z}, \alpha_{1}^{*}\left(G_{n}\right), G_{n}\right)+\delta \beta^{2} / 8-2 \epsilon \\
& >H_{1}\left(\mathbf{Z}, \alpha_{1}^{*}\left(G_{n}\right), G_{n}\right),
\end{aligned}
$$

which contradicts the fact that $H_{1}\left(\mathbf{Z}, \breve{\alpha}_{1}^{*}\left(G_{n}\right), G_{n}\right)$ is the minimum of $H_{1}\left(\mathbf{Z}, \alpha, G_{n}\right)$.

The proof of Theorem $6(i i)$ is skipped since it is similar.

\section{References}

Abramowitz, M. and Stegun, I. (1972). Handbook of mathematical functions, Dover, New York.

Baldi, P. and Long, A. D. (2001). A bayesian framework for the analysis of microarray expression data: regularized $t$-test and statistical inferences of gene changes, Bioinformatics pp. 509519 .

Brewster, J. F. and Zidek, J. V. (1974). Improving on equivariant estimators, Annals of Statistics 2: 21-38.

Brown, L. (1968). Inadmissibility of the usual estimators of scale parameters in problems with unknown location and scale parameters, Ann. Math. Statist. 39: 29-48.

Callow, M. J., Dudoit, S., Gong, E. L., Speed, T. P. and Rubin, E. M. (2000). Microarray expression profiling identifies genes with altered expression in hdl-deficient mice, Genome Research 10: 2022-2029.

Cui, X. and Churchill, G. A. (2003). Statistical tests for differential expression in cDNA microarray experiments, Genome Biology.

Cui, X., Hwang, J. T. G., Qiu, J., Blades, N. J. and Churchill, G. A. (2005). Improved statistical tests for differential gene expression by shrinking variance components estimates, Biostatistics 6: 59-75. 
Ferguson, T. S. (1996). A course in large sample theory, London: Chapman and Hall.

Jain, N., Thatte, J., Braciale, T., Ley, K., O'Connell, M. and Lee, J. (2003). Local-pooled error test for identifying differentially expressed genes with a small number of replicated microarrays, Bioinformatics 19: 1945-1951.

James, W. and Stein, C. (1961). Estimation with quadratic loss, Proc. 4th Berkeley Sympos. Math. Statist. and Prob. 1: 361-379.

Kamb, A. and Ramaswami, A. (2001). A simple method for statistical analysis of intensity differences in microarray-derived gene expression data, BMC Biotechnol. pp. 1-8.

Kendziorski, C. M., Newton, M. A., Lan, H. and Could, M. N. (2003). On parametric empirical bayes methods for comparing multiple groups using replicated gene expression profiles, Statistics in Medicine 22: 3899-3914.

Kerr, M. K., Afshari, C. A., Bennett, L., Bushel, P., Martinez, J., Walker, N. J. and Churchill, G. A. (2002). Statistical analysis of a gene expression microarray experiment with replication, Statistica Sinica 12: 203-218.

Kubokawa, T. (1994). A unified approach to improving equivariant estimators, Annals of Statistics 22: 290-299.

Kubokawa, T. (1999). Shrinkage and modification techniques in estimation of variance and the related problems: A review, Comm. Statist. A-Theory Methods 28: 613-650.

Kubokawa, T. and Srivastava, M. S. (2003). Estimating the covariance matrix: a new approach, Journal of Multivariate Analysis 86: 28-47.

Ledoit, O. and Wolf, M. (2004a). Honey, I shrunk the sample covariance matrix, Journal of Portfolio Management 30: 110-119.

Ledoit, O. and Wolf, M. (2004b). A well-conditioned estimator for large-dimensional covariance matrices, Journal of Multivariate Analysis 88: 365-411.

Leung, Y. and Cavalieri, D. (2003). Fundamentals of cDNA microarray data analysis, TRENDS in Genetics 11: 649-659.

Lin, Y., Nadler, S. T., Attie, A. D. and Yandell, B. S. (2003). Adaptive gene picking with microarray data: detecting important low abundance signals. in Parmigiani, G., Garrett, E. S., Irizarry, R. A. and Zeger, S. L. (ed.), The Analysis of Gene Expression Data: Methods and Software. New York: Springer.

Lindley, D. V. (1962). Discussion of professor stein's paper: Confidence sets for the mean of a multivariate normal distribution, Journal of the Royal Statistical Society B 24: 265-296. 
Littell, R. C., Milliken, G., Stroup, W. W. and Wolfinger, R. D. (1996). SAS System for Mixed Models, SAS Institute Inc., NC.

Lönnstedt, I. and Speed, T. (2002). Replicated microarray data, Statistica Sinica 12: 31-46.

Maatta, J. M. and Casella, G. (1990). Developments in decision-theoretic variance estimation, Statistical Science 5: 90-101.

Nguyen, D. V., Arpat, A. B., Wang, N. and Carroll, R. J. (2002). DNA microarray experiments: biological and technological aspects, Biometrics 58: 701-717.

Perron, F. (1990). Equivariant estimators of a covariance matrix, Canad. J. Statist. 18: 179182.

Searle, S. R., Casella, G. and McCulloch, C. E. (1992). Variance Components, Wiley, New York.

Sinha, B. K. and Ghosh, M. (1987). Inadmissibility of the best equivariant estimators of the variance-covariance matrix, the precision matrix and the generalized variance under entropy loss, Statist. Decisions 5: 201-227.

Stein, C. (1964). Inadmissibility of the usual estimator for the variance of a normal distribution with unknown mean, Ann. Inst. Statist. Math. 16: 155-160.

Storey, J. and Tibshirani, R. (2003). SAM thresholding and false discovery rates for detecting differential gene expression in DNA microarrays. in Parmigiani, G., Garrett, E. S., Irizarry, R. A. and Zeger, S. L. (ed.), The Analysis of Gene Expression Data: Methods and Software. New York: Springer.

Wright, G. W. and Simon, R. M. (2003). A random variance model for detection of differential gene expression in small microarray experiments, Bioinformatics 19: 2448-2455.

Wu, H. and Churchill, G. (2005). R/MAANOVA: an extensive R environment for the analysis of microarray experiments, http://www.jax.org/staff/churchill/labsite/software/Rmaanova/maanova.pdf. 